\title{
GEOMETRY OF THE $L_{q}$-CENTROID BODIES OF AN ISOTROPIC LOG-CONCAVE MEASURE
}

\author{
APOSTOLOS GIANNOPOULOS, PANTELIS STAVRAKAKIS, ANTONIS TSOLOMITIS, \\ AND BEATRICE-HELEN VRITSIOU
}

\begin{abstract}
We study some geometric properties of the $L_{q}$-centroid bodies $Z_{q}(\mu)$ of an isotropic log-concave measure $\mu$ on $\mathbb{R}^{n}$. For any $2 \leqslant q \leqslant \sqrt{n}$ and for $\varepsilon \in\left(\varepsilon_{0}(q, n), 1\right)$ we determine the inradius of a random $(1-\varepsilon) n$-dimensional projection of $Z_{q}(\mu)$ up to a constant depending polynomially on $\varepsilon$. Using this fact we obtain estimates for the covering numbers $N\left(\sqrt{q} B_{2}^{n}, t Z_{q}(\mu)\right), t \geqslant 1$, thus showing that $Z_{q}(\mu)$ is a $\beta$-regular convex body. As a consequence, we also get an upper bound for $M\left(Z_{q}(\mu)\right)$.
\end{abstract}

\section{INTRODUCTION}

Given a convex body $K$ of volume 1 or a log-concave probability measure $\mu$ on $\mathbb{R}^{n}$, we define the $L_{q}$-centroid bodies $Z_{q}(K)$ or $Z_{q}(\mu), q \in(0,+\infty)$, through their support function $h_{Z_{q}(K)}$ or $h_{Z_{q}(\mu)}$, which is defined as follows: for every $y \in \mathbb{R}^{n}$,

$$
\begin{gathered}
h_{Z_{q}(K)}(y):=\|\langle\cdot, y\rangle\|_{L_{q}(K)}=\left(\int_{K}|\langle x, y\rangle|^{q} d x\right)^{1 / q} \\
h_{Z_{q}(\mu)}(y):=\|\langle\cdot, y\rangle\|_{L_{q}(\mu)}=\left(\int_{\mathbb{R}^{n}}|\langle x, y\rangle|^{q} d \mu(x)\right)^{1 / q} .
\end{gathered}
$$

These bodies then incorporate information about the distribution of linear functionals with respect to the uniform measure on $K$ or with respect to the probability measure $\mu$. The $L_{q}$-centroid bodies were introduced, under a different normalization, by Lutwak, Yang and Zhang in 25, while in 30. for the first time, and in 31] later on, Paouris used geometric properties of them to acquire detailed information about the distribution of the Euclidean norm with respect to the uniform measure on isotropic convex bodies. An asymptotic theory for the $L_{q}$-centroid bodies has since been developed in the context of isotropic measures, and it seems to advance in parallel with all recent developments in the area.

Recall that a convex body $K$ in $\mathbb{R}^{n}$ is called isotropic if it has volume 1 , it is centered, i.e. its barycenter is at the origin, and its inertia matrix is a multiple of the identity matrix: there exists a constant $L_{K}>0$ such that

$$
\int_{K}\langle x, \theta\rangle^{2} d x=L_{K}^{2}
$$

for every $\theta$ in the Euclidean unit sphere $S^{n-1}$. Similarly, a log-concave probability measure $\mu$ on $\mathbb{R}^{n}$ is called isotropic if its barycenter is at the origin and if its inertia

Received by the editors January 21, 2013.

2010 Mathematics Subject Classification. Primary 52A23; Secondary 46B06, 52A40, 60D05. 
matrix is the identity matrix; in that case the isotropic constant of the measure is defined as

$$
L_{\mu}:=\sup _{x \in \mathbb{R}^{n}}\left(f_{\mu}(x)\right)^{1 / n},
$$

where $f_{\mu}$ is the density of $\mu$ with respect to the Lebesgue measure. One very well-known open question in the theory of isotropic measures is the hyperplane conjecture, which asks if there exists an absolute constant $C>0$ such that

$$
L_{n}:=\max \left\{L_{K}: K \text { is isotropic in } \mathbb{R}^{n}\right\} \leqslant C
$$

for all $n \geqslant 1$. Bourgain proved in [4] that $L_{n} \leqslant c \sqrt[4]{n} \log n$, while Klartag [18] obtained the bound $L_{n} \leqslant c \sqrt[4]{n}$. A second proof of Klartag's bound appears in [19].

A motivation for this paper is a recent reduction [16] of the hyperplane conjecture to the study of geometric properties of the $L_{q}$-centroid bodies, and in particular to the study of the parameter

$$
I_{1}\left(K, Z_{q}^{\circ}(K)\right):=\int_{K}\|x\|_{Z_{q}^{\circ}(K)}(x) d x=\int_{K} h_{Z_{q}(K)}(x) d x .
$$

The main result of [16] is, in a sense, a continuation of Bourgain's approach to the problem and, roughly speaking, can be formulated as follows: Given $q \geqslant 2$ and $\frac{1}{2} \leqslant s \leqslant 1$, an upper bound of the form $I_{1}\left(K, Z_{q}^{\circ}(K)\right) \leqslant C_{1} q^{s} \sqrt{n} L_{K}^{2}$ for all isotropic convex bodies $K$ in $\mathbb{R}^{n}$ leads to the estimate

$$
L_{n} \leqslant \frac{C_{2} \sqrt[4]{n} \log n}{q^{\frac{1-s}{2}}} .
$$

Bourgain's estimate may be recovered by choosing $q=2$; however, clarifying the behaviour of $I_{1}\left(K, Z_{q}^{\circ}(K)\right)$ might allow one to use much larger values of $q$. This behaviour is most naturally related to the geometry of the bodies $Z_{q}(K)$ and especially how this geometry is affected by or affects the geometry of the body $K$. This is not yet fully understood, and, in view of (1.6), we believe that its deeper study would be very useful.

In Section 3 we give an account of some basic known results for the bodies $Z_{q}(K)$ and, more generally, the bodies $Z_{q}(\mu)$ where $\mu$ is an isotropic log-concave measure on $\mathbb{R}^{n}$. In the range $2 \leqslant q \leqslant \sqrt{n}$, for example, some of their global parameters are completely determined: the volume radius and the mean width of $Z_{q}(\mu)$ are of the same order $\sqrt{q}$. The purpose of this work is to provide new information on the local structure of $Z_{q}(\mu)$, which in turn has some interesting consequences. Our first main result concerns proportional projections of the centroid bodies.

Theorem 1.1. Let $\mu$ be an isotropic log-concave measure on $\mathbb{R}^{n}$. Fix $1 \leqslant \alpha<2$. For every $0<\varepsilon<1$ and any $q \leqslant \sqrt{\varepsilon n}$ there are $k \geqslant(1-\varepsilon) n$ and $F \in G_{n, k}$ such that

$$
P_{F}\left(Z_{q}(\mu)\right) \supseteq c(2-\alpha) \varepsilon^{\frac{1}{2}+\frac{2}{\alpha}} \sqrt{q} B_{F},
$$

where $c>0$ is an absolute constant (independent of $\alpha, \varepsilon$, the measure $\mu, q$ or $n$ ). Moreover, for any $2 \leqslant q \leqslant \varepsilon n$ there are $k \geqslant(1-\varepsilon) n$ and $F \in G_{n, k}$ such that

$$
P_{F}\left(Z_{q}(\mu)\right) \supseteq \frac{c_{1}(2-\alpha) \varepsilon^{\frac{1}{2}+\frac{2}{\alpha}}}{L_{\varepsilon n}} \sqrt{q} B_{F} \supseteq \frac{c_{2}(2-\alpha) \varepsilon^{\frac{1}{4}+\frac{2}{\alpha}}}{\sqrt[4]{n}} \sqrt{q} B_{F},
$$

where $c_{1}, c_{2}>0$ are absolute constants. 
The proof of Theorem 1.1 is given in Section 5. We use Pisier's theorem on the existence of $\alpha$-regular ellipsoids for symmetric convex bodies; we combine this with available information on the $L_{q}$-centroid bodies as well as results from [1] concerning the circumradius of proportional sections of $\alpha$-regular convex bodies, the proofs of which are outlined in Section 4. Let us mention that the dual result is a direct consequence of the low $M^{*}$-estimate, since the mean width of $Z_{q}(\mu)$ is known to be of the order of $\sqrt{q}$ : if $2 \leqslant q \leqslant \sqrt{n}$ and if $\varepsilon \in(0,1)$ and $k=(1-\varepsilon) n$, then a subspace $F \in G_{n, k}$ satisfies

$$
P_{F}\left(Z_{q}^{\circ}(\mu)\right) \supseteq \frac{c_{1} \sqrt{\varepsilon}}{\sqrt{q}} B_{F}
$$

with probability greater than $1-\exp \left(-c_{2} \varepsilon n\right)$, where $c_{1}, c_{2}>0$ are absolute constants.

In Section 6 we discuss bounds for the covering numbers of a Euclidean ball by $Z_{q}(\mu)$. It was proved in [14] and [15] that if $\mu$ is an isotropic log-concave measure on $\mathbb{R}^{n}$, then for any $1 \leqslant q \leqslant n$ and $t \geqslant 1$,

$$
\log N\left(Z_{q}(\mu), c_{1} t \sqrt{q} B_{2}^{n}\right) \leqslant c_{2} \frac{n}{t^{2}}+c_{3} \frac{\sqrt{q n}}{t},
$$

where $c_{1}, c_{2}, c_{3}>0$ are absolute constants. Using Theorem 1.1 and an entropy extension result from [24] we obtain regular entropy estimates for the dual covering numbers.

Theorem 1.2. Let $\mu$ be an isotropic log-concave measure on $\mathbb{R}^{n}$. Assume $1 \leqslant \alpha<$ 2. Then, for any $q \leqslant \sqrt{n}$ and any

$$
1 \leqslant t \leqslant \min \left\{\sqrt{q}, c_{1}(2-\alpha)^{-1}\left(n / q^{2}\right)^{\frac{\alpha+4}{2 \alpha}}\right\}
$$

we have

$$
\log N\left(\sqrt{q} B_{2}^{n}, t Z_{q}(\mu)\right) \leqslant c(\alpha) \frac{n}{t^{\frac{2 \alpha}{\alpha+4}}} \max \left\{\log \frac{\sqrt{2 q}}{t}, \log \frac{1}{(2-\alpha) t}\right\},
$$

where $c(\alpha) \leqslant C(2-\alpha)^{-2 / 3}$ and $c_{1}, C$ are absolute constants. Moreover, for any $2 \leqslant q \leqslant n$ and any

$$
1 \leqslant t \leqslant \min \left\{\sqrt{q}, c_{2}(2-\alpha)^{-1} L_{n}\left(\frac{n}{q}\right)^{\frac{\alpha+4}{2 \alpha}}\right\}
$$

we have

$$
\log N\left(\sqrt{q} B_{2}^{n}, t Z_{q}(\mu)\right) \leqslant c(\alpha) L_{n}^{\frac{2 \alpha}{\alpha+4}} \frac{n}{t^{\frac{2 \alpha}{\alpha+4}}} \max \left\{\log \frac{2 q}{t^{2}}, \log \frac{L_{n}}{(2-\alpha) t}\right\},
$$

where $c(\alpha)$ is as above and $c_{2}$ is an absolute constant.

Note that since $Z_{q}(\mu) \supseteq B_{2}^{n}$, we are interested in bounds for the above covering numbers when $t$ is in the interval $[1, \sqrt{q}]$. An analysis of the restrictions in Theorem 1.2 shows that, given any $q \leqslant n^{3 / 7}$, (1.11) holds true with any $t$ in the "interesting" interval, while the same is true for (1.12) as long as $q \leqslant \sqrt{L_{n}} n^{3 / 4}$. Although all these estimates are most probably not optimal, we can still conclude that $Z_{q}(\mu)$, with $q \leqslant n^{3 / 7}$, is a $\beta$-regular convex body in the sense of Pisier's theorem (for some concrete positive value of $\beta$ ). As a consequence of this fact we get an upper bound for the parameter

$$
M\left(Z_{q}(\mu)\right)=\int_{S^{n-1}}\|x\|_{Z_{q}(\mu)} d \sigma(x) .
$$


Recall that the dual Sudakov inequality of Pajor and Tomczak-Jaegermann (see e.g. 33] ) provides 2-regular entropy estimates for the numbers $N\left(B_{2}^{n}, t C\right)$ in terms of $M(C)$; namely, it shows that

$$
\log N\left(B_{2}^{n}, t C\right) \leqslant c n\left(\frac{M(C)}{t}\right)^{2}
$$

for every $t \geqslant 1$. In Section 7 we use in a converse manner the entropy estimates of Theorem 1.2 to obtain non-trivial upper bounds for $M\left(Z_{q}(\mu)\right)$.

Theorem 1.3. Let $\mu$ be an isotropic log-concave measure on $\mathbb{R}^{n}$. For every $1 \leqslant$ $q \leqslant n^{3 / 7}$,

$$
M\left(Z_{q}(\mu)\right) \leqslant C \frac{(\log q)^{5 / 6}}{\sqrt[6]{q}} .
$$

Moreover, for every $q$ such that $L_{n}^{2} \log ^{2} q \leqslant q \leqslant \sqrt{L_{n}} n^{3 / 4}$,

$$
M\left(Z_{q}(\mu)\right) \leqslant C \frac{\sqrt[3]{L_{n}}(\log q)^{5 / 6}}{\sqrt[6]{q}} .
$$

Observe now that if $K$ is an isotropic convex body in $\mathbb{R}^{n}$ with isotropic constant $L_{K}$, then the measure $\mu_{K}$ with density $f_{\mu_{K}}(x):=L_{K}^{n} \mathbf{1}_{K / L_{K}}(x)$ is isotropic and, for every $q>0$, it holds that $Z_{q}(K)=L_{K} Z_{q}\left(\mu_{K}\right)$. Using also the fact that $M(K) \leqslant M\left(Z_{q}(K)\right)$ for every symmetric convex body $K$ and every $q>0$, we can use the above bounds for $M\left(Z_{q}\left(\mu_{K}\right)\right)$ to obtain an upper bound for $M(K)$ in the isotropic case.

Theorem 1.4. Let $K$ be a symmetric isotropic convex body in $\mathbb{R}^{n}$. Then,

$$
M(K) \leqslant C \frac{\sqrt[4]{L_{n}}(\log n)^{5 / 6}}{L_{K} \sqrt[8]{n}} .
$$

This is a question that until recently had not attracted much attention. Valettas, using a slightly different approach [35], has shown that

$$
M(K) \leqslant \frac{C(\log n)^{1 / 3}}{\sqrt[12]{n} L_{K}}
$$

for every symmetric isotropic convex body $K$ in $\mathbb{R}^{n}$, where $C>0$ is an absolute constant. Note that, on the other hand, there are many approaches concerning the corresponding question about the mean width that give the best currently known estimate:

$$
w(K) \leqslant C n^{3 / 4} L_{K}
$$

for every isotropic convex body $K$ in $\mathbb{R}^{n}$. Nevertheless, this problem as well remains open (for a discussion about it, see [15] and the references therein).

We close this paper with some additional observations on the geometry of the centroid bodies $Z_{q}(\mu)$ and their polars. We first provide lower bounds for the radius of their sections; actually, they hold true for every $1 \leqslant k<n$ and any $F \in G_{n, k}$. By duality, these estimates (combined with e.g. Proposition 5.3 ) determine the inradius of their random projections. We also provide upper bounds for the parameters $M_{-k}\left(Z_{q}(\mu)\right)$ and $I_{-k}\left(\overline{Z_{q}}(\mu)\right)$ (see Section 8 for the precise definitions). These imply small ball probability estimates for the Euclidean norm on $Z_{q}(\mu)$. All the results are based on the main estimates from Section 5 and Section 6. Although they are not optimal, we describe our approach and sketch their proofs; we expect that further progress can be achieved along the same lines. 


\section{Notation AND PRELiminaries}

We work in $\mathbb{R}^{n}$, which is equipped with a Euclidean structure $\langle\cdot, \cdot\rangle$. We denote the corresponding Euclidean norm by $\|\cdot\|_{2}$ and write $B_{2}^{n}$ for the Euclidean unit ball and $S^{n-1}$ for the unit sphere. Volume is denoted by $|\cdot|$. We write $\omega_{n}$ for the volume of $B_{2}^{n}$ and $\sigma$ for the rotationally invariant probability measure on $S^{n-1}$. We also denote the Haar measure on $O(n)$ by $\nu$. The Grassmann manifold $G_{n, k}$ of $k$-dimensional subspaces of $\mathbb{R}^{n}$ is equipped with the Haar probability measure $\nu_{n, k}$. Let $k \leqslant n$ and $F \in G_{n, k}$. We will denote the orthogonal projection from $\mathbb{R}^{n}$ onto $F$ by $P_{F}$. We also define $B_{F}:=B_{2}^{n} \cap F$ and $S_{F}:=S^{n-1} \cap F$.

The letters $c, c^{\prime}, c_{1}, c_{2}$, etc., denote absolute positive constants whose value may change from line to line. Whenever we write $a \simeq b$, we mean that there exist absolute constants $c_{1}, c_{2}>0$ such that $c_{1} a \leqslant b \leqslant c_{2} a$. Also, if $K, L \subseteq \mathbb{R}^{n}$ we will write $K \simeq L$ if there exist absolute constants $c_{1}, c_{2}>0$ such that $c_{1} K \subseteq L \subseteq c_{2} K$.

Convex bodies. A convex body in $\mathbb{R}^{n}$ is a compact convex subset $C$ of $\mathbb{R}^{n}$ with non-empty interior. We say that $C$ is symmetric if $x \in C$ implies that $-x \in C$. We say that $C$ is centered if the barycenter of $C$ is at the origin, i.e. $\int_{C}\langle x, \theta\rangle d x=0$ for every $\theta \in S^{n-1}$. The volume radius of $C$ is the quantity

$$
\operatorname{vrad}(C)=\left(\frac{|C|}{\left|B_{2}^{n}\right|}\right)^{1 / n} .
$$

The support function of a convex body $C$ is defined by

$$
h_{C}(y):=\max \{\langle x, y\rangle: x \in C\}
$$

and characterizes $C$ uniquely. The mean width of $C$ is

$$
w(C):=\int_{S^{n-1}} h_{C}(\theta) \sigma(d \theta)
$$

and the radius of $C$ is the quantity $R(C):=\max \left\{\|x\|_{2}: x \in C\right\}$. Also, if the origin is an interior point of $C$, the polar body $C^{\circ}$ of $C$ is defined as follows:

$$
C^{\circ}:=\left\{y \in \mathbb{R}^{n}:\langle x, y\rangle \leqslant 1 \text { for all } x \in C\right\} .
$$

Let $C \subset \mathbb{R}^{n}$ be a symmetric convex body. We write $\|\cdot\|_{C}$ for the norm $\|x\|_{C}=$ $\min \{t \geqslant 0: x \in t C\}$ induced to $\mathbb{R}^{n}$ by $C$, and we define

$$
M(C):=\int_{S^{n-1}}\|\theta\|_{C} d \sigma(\theta) .
$$

Note that $M(C)=w\left(C^{\circ}\right)$ and that

$$
M(C)^{-1} \leqslant \operatorname{vrad}(C) \leqslant w(C)=M\left(C^{\circ}\right) ;
$$

the left hand side inequality is easily checked if we express the volume of $C$ as an integral in polar coordinates and use Hölder's and Jensen's inequalities, while the right hand side inequality is the classical Urysohn's inequality. We also need Milman's low $M^{*}$-estimate (see [29] or [33] for precise references): if $C$ is a symmetric convex body in $\mathbb{R}^{n}$, then a subspace $F \in G_{n, k}$ satisfies

$$
R(C \cap F) \leqslant c_{1} \sqrt{\frac{n}{n-k}} w(C)
$$

with probability greater than $1-\exp \left(-c_{2}(n-k)\right)$, where $c_{1}, c_{2}>0$ are absolute constants. 
Furthermore, if $C$ is a symmetric convex body in $\mathbb{R}^{n}$, we define $k_{*}(C)$ to be the largest positive integer $k \leqslant n$ with the property that the measure $\nu_{n, k}$ of $F \in G_{n, k}$ for which we have $\frac{1}{2} w(C) B_{F} \subseteq P_{F}(C) \subseteq 2 w(C) B_{F}$ is greater than $\frac{n}{n+k}$. It is known that this parameter is completely determined by the dimension, the mean width and the radius of $C$ :

$$
c_{3} n \frac{w(C)^{2}}{R(C)^{2}} \leqslant k_{*}(C) \leqslant c_{4} n \frac{w(C)^{2}}{R(C)^{2}} .
$$

Recall that the covering number $N(A, B)$ of a body $A$ by a second body $B$ is the least integer $N$ for which there exist $N$ translates of $B$ whose union covers $A$. Milman (see e.g. 26]) proved that there exists an absolute constant $\beta>0$ such that every centered convex body $K$ in $\mathbb{R}^{n}$ has a linear image $\tilde{K}$ which satisfies $|\tilde{K}|=\left|B_{2}^{n}\right|$ and

$$
\max \left\{N\left(\tilde{K}, B_{2}^{n}\right), N\left(B_{2}^{n}, \tilde{K}\right), N\left(\tilde{K}^{\circ}, B_{2}^{n}\right), N\left(B_{2}^{n}, \tilde{K}^{\circ}\right)\right\} \leqslant \exp (\beta n) .
$$

We say that a convex body $K$ which satisfies this estimate is in $M$-position with constant $\beta$.

Pisier 32 has proposed a different approach to this result, which allows one to find a whole family of $M$-ellipsoids and to give more detailed information on the behaviour of the corresponding covering numbers. The precise statement is as follows.

Theorem 2.1 (Pisier). For every $0<\alpha<2$ and every symmetric convex body $K$ in $\mathbb{R}^{n}$ there exists a linear image $\tilde{K}$ of $K$ such that

$$
\max \left\{N\left(\tilde{K}, t B_{2}^{n}\right), N\left(B_{2}^{n}, t \tilde{K}\right), N\left(\tilde{K}^{\circ}, t B_{2}^{n}\right), N\left(B_{2}^{n}, t \tilde{K}^{\circ}\right)\right\} \leqslant \exp \left(\frac{c(\alpha) n}{t^{\alpha}}\right)
$$

for every $t \geqslant 1$, where $c(\alpha)$ depends only on $\alpha$, and $c(\alpha)=O\left((2-\alpha)^{-\alpha / 2}\right)$ as $\alpha \rightarrow 2$.

For basic facts from the Brunn-Minkowski theory and the asymptotic theory of finite dimensional normed spaces whose unit balls are various symmetric convex bodies appearing in this paper, we refer to the books [34, 29] and [33].

Log-concave probability measures. We denote by $\mathcal{P}_{n}$ the class of all Borel probability measures on $\mathbb{R}^{n}$ which are absolutely continuous with respect to the Lebesgue measure. The density of $\mu \in \mathcal{P}_{n}$ is denoted by $f_{\mu}$. We say that $\mu \in \mathcal{P}_{n}$ is centered and we write $\operatorname{bar}(\mu)=0$ if for all $\theta \in S^{n-1}$,

$$
\int_{\mathbb{R}^{n}}\langle x, \theta\rangle d \mu(x)=\int_{\mathbb{R}^{n}}\langle x, \theta\rangle f_{\mu}(x) d x=0 .
$$

A measure $\mu$ on $\mathbb{R}^{n}$ is called log-concave if $\mu(\lambda A+(1-\lambda) B) \geqslant \mu(A)^{\lambda} \mu(B)^{1-\lambda}$ for any compact subsets $A$ and $B$ of $\mathbb{R}^{n}$ and any $\lambda \in(0,1)$. A function $f: \mathbb{R}^{n} \rightarrow[0, \infty)$ is called log-concave if its support $\{f>0\}$ is a convex set and the restriction of $\log f$ to it is concave. It is known that if a probability measure $\mu$ is log-concave and $\mu(H)<1$ for every hyperplane $H$, then $\mu \in \mathcal{P}_{n}$ and its density $f_{\mu}$ is log-concave. Note that if $K$ is a convex body in $\mathbb{R}^{n}$, then the Brunn-Minkowski inequality implies that $\mathbf{1}_{K}$ is the density of a log-concave measure. 
If $\mu$ is a log-concave measure on $\mathbb{R}^{n}$ with density $f_{\mu}$, we define the isotropic constant of $\mu$ by

$$
L_{\mu}:=\left(\frac{\sup _{x \in \mathbb{R}^{n}} f_{\mu}(x)}{\int_{\mathbb{R}^{n}} f_{\mu}(x) d x}\right)^{\frac{1}{n}}[\operatorname{det} \operatorname{Cov}(\mu)]^{\frac{1}{2 n}},
$$

where $\operatorname{Cov}(\mu)$ is the covariance matrix of $\mu$ with entries

$$
\operatorname{Cov}(\mu)_{i j}:=\frac{\int_{\mathbb{R}^{n}} x_{i} x_{j} f_{\mu}(x) d x}{\int_{\mathbb{R}^{n}} f_{\mu}(x) d x}-\frac{\int_{\mathbb{R}^{n}} x_{i} f_{\mu}(x) d x}{\int_{\mathbb{R}^{n}} f_{\mu}(x) d x} \frac{\int_{\mathbb{R}^{n}} x_{j} f_{\mu}(x) d x}{\int_{\mathbb{R}^{n}} f_{\mu}(x) d x} .
$$

We say that a log-concave probability measure $\mu$ on $\mathbb{R}^{n}$ is isotropic if $\operatorname{bar}(\mu)=0$ and $\operatorname{Cov}(\mu)$ is the identity matrix, and we write $\mathcal{I} \mathcal{L}_{n}$ for the class of isotropic logconcave probability measures on $\mathbb{R}^{n}$. Note that a centered convex body $K$ of volume 1 in $\mathbb{R}^{n}$ is isotropic; i.e. it satisfies (1.2) if and only if the log-concave probability measure $\mu_{K}$ with density $x \mapsto L_{K}^{n} \mathbf{1}_{K / L_{K}}(x)$ is isotropic.

Let $\mu \in \mathcal{P}_{n}$. For every $1 \leqslant k \leqslant n-1$ and every $E \in G_{n, k}$, the marginal of $\mu$ with respect to $E$ is the probability measure with density

$$
f_{\pi_{E} \mu}(x)=\int_{x+E^{\perp}} f_{\mu}(y) d y .
$$

It is easily checked that if $\mu$ is centered, isotropic or log-concave, then $\pi_{E} \mu$ is also centered, isotropic or log-concave, respectively.

For more information on isotropic convex bodies and log-concave measures see [28], 2] and [9].

\section{3. $L_{q}$-CENTROID BODIES: BASIC FACTS}

Recall that if $\mu$ is a log-concave probability measure on $\mathbb{R}^{n}$, the $L_{q}$-centroid body $Z_{q}(\mu), q \geqslant 1$, of $\mu$ is the centrally symmetric convex body with support function

$$
h_{Z_{q}(\mu)}(y):=\left(\int_{\mathbb{R}^{n}}|\langle x, y\rangle|^{q} d \mu(x)\right)^{1 / q} .
$$

Observe that $\mu$ is isotropic if and only if it is centered and $Z_{2}(\mu)=B_{2}^{n}$. From Hölder's inequality it follows that $Z_{1}(\mu) \subseteq Z_{p}(\mu) \subseteq Z_{q}(\mu)$ for all $1 \leqslant p \leqslant q<\infty$. Conversely, using Borel's lemma (see [29, Appendix III]), one can check that

$$
Z_{q}(\mu) \subseteq c \frac{q}{p} Z_{p}(\mu)
$$

for all $1 \leqslant p<q$. In particular, if $\mu$ is isotropic, then $R\left(Z_{q}(\mu)\right) \leqslant c q$.

As we saw in the previous section, if $K$ is a convex body of volume $1 \mathrm{in} \mathbb{R}^{n}$, then the measure with density $x \mapsto \mathbf{1}_{K}(x)$ is a log-concave probability measure on $\mathbb{R}^{n}$ and the $L_{q}$-centroid bodies $Z_{q}(K)$ of $K$ can be defined as above. By Hölder's inequality we again have $Z_{1}(K) \subseteq Z_{p}(K) \subseteq Z_{q}(K) \subseteq \operatorname{conv}\{K,-K\}$ for all $1 \leqslant p \leqslant q<\infty$, so if $K$ is symmetric, then $Z_{p}(K) \subseteq K$ for every $p<\infty$.

Using Fubini's theorem we see that for every $1 \leqslant k \leqslant n-1$ and every $F \in G_{n, k}$ and $q \geqslant 1$,

$$
P_{F}\left(Z_{q}(\mu)\right)=Z_{q}\left(\pi_{F}(\mu)\right)
$$

In [30] Paouris shows that the moments

$$
I_{q}(\mu):=\left(\int_{\mathbb{R}^{n}}\|x\|_{2}^{q} d x\right)^{1 / q}, \quad q \in(-n,+\infty) \backslash\{0\},
$$


of the Euclidean norm with respect to an isotropic log-concave probability measure $\mu$ on $\mathbb{R}^{n}$ remain comparable to $I_{2}(\mu)=\sqrt{n}$ when $q \geqslant 2$ does not exceed a parameter $q_{*}(\mu)$ of the measure that he defines as follows:

$$
q_{*}(\mu):=\max \left\{q \leqslant n: k_{*}\left(Z_{q}(\mu)\right) \geqslant q\right\} .
$$

He proves this by showing that

$$
I_{q}(\mu) \simeq \sqrt{\frac{n}{q}} w\left(Z_{q}(\mu)\right)
$$

and that

$$
w\left(Z_{q}(\mu)\right) \simeq \sqrt{q}
$$

for every $q \leqslant q_{*}(\mu)$. He establishes that

$$
q_{*}(\mu) \geqslant c_{1} \sqrt{n}
$$

for every isotropic measure $\mu$ on $\mathbb{R}^{n}$, where $c_{1}>0$ is an absolute constant and shows that there are isotropic measures $\mu$ on $\mathbb{R}^{n}$ such that $q_{*}(\mu) \simeq \sqrt{n}$. Furthermore, he proves that

$$
I_{q}(\mu) \simeq R\left(Z_{q}(\mu)\right)
$$

for every $q \in\left[q_{*}(\mu), n\right]$, and he gives an upper bound for the volume radius of the $L_{q}$-centroid bodies:

$$
\left|Z_{q}(\mu)\right|^{1 / n} \leqslant c_{2} \sqrt{q / n}
$$

for all $1 \leqslant q \leqslant n$, where $c_{2}$ is an absolute constant.

In 31] Paouris extends his approach to describe the behaviour of the negative moments of the Euclidean norm with respect to an isotropic measure $\mu$ on $\mathbb{R}^{n}$. He shows that

$$
I_{-q}(\mu) \simeq I_{2}(\mu)=\sqrt{n} \text { for all } 0<q \leqslant q_{*}(\mu) .
$$

However, unlike the positive moments $I_{q}(\mu)$ that, as we saw, do not remain comparable to $I_{2}(\mu)$ once $q$ gets larger than $q_{*}(\mu)$, the behaviour of the corresponding negative moments is not known and, in fact, (3.10) may hold with any positive $q$ up to $n-1$. This question actually is equivalent to the hyperplane conjecture, as Dafnis and Paouris proved in [8] by introducing another parameter that for each $\delta \geqslant 1$ is given by

$$
q_{-c}(\mu, \delta):=\max \left\{1 \leqslant q \leqslant n-1: I_{-q}(\mu) \geqslant \delta^{-1} I_{2}(\mu)=\delta^{-1} \sqrt{n}\right\} ;
$$

namely this measures how large the range of $(3.10)$ is if we allow the implied constants to depend on $\delta$. Dafnis and Paouris established that

$$
L_{n} \leqslant C \delta \sup _{\mu \in \mathcal{I} L_{[n]}} \sqrt{\frac{n}{q_{-c}(\mu, \delta)}} \log \left(\frac{e n}{q_{-c}(\mu, \delta)}\right)
$$

for every $\delta \geqslant 1$, and they also showed that if the hyperplane conjecture is correct, that is, if (1.4) holds true, then we will have

$$
q_{-c}\left(\mu, \delta_{0}\right)=n-1
$$


for some $\delta_{0} \simeq 1$, for every isotropic log-concave measure $\mu$ on $\mathbb{R}^{n}$. Note that by (3.10), we already know that

$$
q_{-c}\left(\mu, \delta_{1}\right) \geqslant q_{*}(\mu) \geqslant c_{1} \sqrt{n}
$$

where $\delta_{1} \geqslant 1$ and $c_{1}>0$ are absolute constants.

Next we turn to lower bounds for the volume radius of the $L_{q}$-centroid bodies of measures $\mu \in \mathcal{I} \mathcal{L}_{[n]}$. From [25] we know that for every $1 \leqslant q \leqslant n$,

$$
\left|Z_{q}(\mu)\right|^{1 / n} \geqslant c_{2} \sqrt{q / n} L_{\mu}^{-1}
$$

for some absolute constant $c_{2}>0$. In [19] Klartag and Milman define a "hereditary" variant of $q_{*}(\mu)$ as follows:

$$
q_{*}^{H}(\mu):=n \inf _{k} \inf _{E \in G_{n, k}} \frac{q_{*}\left(\pi_{E} \mu\right)}{k},
$$

where $\pi_{E} \mu$ is the marginal of $\mu$ with respect to $E$, and then, for every $q \leqslant q_{*}^{H}(\mu)$, they give a lower bound for the volume radius of the bodies $Z_{q}(\mu)$ matching the upper bound in (3.9):

$$
\left|Z_{q}(\mu)\right|^{1 / n} \geqslant c_{3} \sqrt{q / n}
$$

where $c_{3}>0$ is an absolute constant. Recall that if $\mu$ is an isotropic log-concave measure, then so are all its marginals; thus, for every subspace $E \in G_{n, k}$, we have by (3.7) that $q_{*}\left(\pi_{E} \mu\right) \geqslant c_{1} \sqrt{k}$. This implies that for all measures $\mu \in \mathcal{I} \mathcal{L}_{[n]}$, $q_{*}^{H}(\mu) \geqslant c_{1} \sqrt{n}$. Note also that for those measures for which we have $q_{*}(\mu) \simeq \sqrt{n}$, the parameter $q_{*}^{H}(\mu)$ as well does not exceed a constant multiple of $\sqrt{n}$. However, the bound (3.17) might hold for larger $q \in[1, n]$ even in the latter case, as was shown by the fourth named author in [37. There a hereditary variant of $q_{-c}(\mu, \delta)$ is introduced as follows:

$$
q_{-c}^{H}(\mu, \delta):=n \inf _{k} \inf _{E \in G_{n, k}} \frac{q_{-c}\left(\pi_{E} \mu, \delta\right)}{k}
$$

for every $\delta \geqslant 1$, and in a similar way as for the bound (3.17) it is established that

$$
\left|Z_{q}(\mu)\right|^{1 / n} \geqslant c_{4} \delta^{-1} \sqrt{q / n}
$$

for every $q \leqslant q_{-c}^{H}(\mu, \delta)$. Of course, we can again see that

$$
q_{-c}^{H}\left(\mu, \delta_{1}\right) \geqslant c_{1} \sqrt{n}
$$

for some $\delta_{1} \simeq 1$ using (3.14) and the definition of $q_{-c}^{H}\left(\mu, \delta_{1}\right)$, but obviously this estimate might be improved if the latter bound were also; such an improvement may be possible but is not trivial, since it would result in better bounds for the isotropic constant problem too, as one can see from (3.12).

To conclude this section, we should stress that in the subsequent proofs we will be using the lower bounds for the volume radius of the $L_{q}$-centroid bodies. However, the only concrete estimate which we currently have for the parameters $q_{*}^{H}(\mu)$ or $q_{-c}^{H}(\mu, \delta)$ and which we can insert in our later estimates is that all of them are at least of the order of $\sqrt{n}$ when $\mu$ is an isotropic log-concave measure on $\mathbb{R}^{n}$. For more information on all the results of this section, see [7]. 


\section{Diameter of Sections of $\alpha$-Regular bodies}

We say that a body $\tilde{K}$ which satisfies the conclusion of Theorem 2.1 is an $\alpha$ regular body. A strong form of the reverse Brunn-Minkowski inequality can be proved for $\alpha$-regular bodies; actually, we only need the regularity of the covering numbers $N\left(K, t B_{2}^{n}\right)$.

Lemma 4.1. Let $\gamma \geqslant 1$, let $\alpha>0$ and let $K_{1}, \ldots, K_{m}$ be symmetric convex bodies in $\mathbb{R}^{n}$ which satisfy

$$
N\left(K_{j}, t B_{2}^{n}\right) \leqslant \exp \left(\frac{\gamma n}{t^{\alpha}}\right)
$$

for all $1 \leqslant j \leqslant m$ and all $t \geqslant 1$. Then,

$$
\left|K_{1}+\cdots+K_{m}\right|^{1 / n} \leqslant C \gamma^{\frac{1}{\alpha}} m^{1+\frac{1}{\alpha}}\left|B_{2}^{n}\right|^{1 / n} .
$$

Proof. We include the very simple proof of this fact. Observe that

$$
\begin{aligned}
N\left(K_{1}+\cdots+K_{m}, t m B_{2}^{n}\right) & =N\left(K_{1}+\cdots+K_{m}, t B_{2}^{n}+\cdots+t B_{2}^{n}\right) \\
& \leqslant \prod_{j=1}^{m} N\left(K_{j}, t B_{2}^{n}\right) \leqslant \exp \left(\gamma n m / t^{\alpha}\right)
\end{aligned}
$$

for all $t \geqslant 1$. It follows that

$$
\left|K_{1}+\cdots+K_{m}\right|^{1 / n} \leqslant t m \exp \left(\gamma m / t^{\alpha}\right)\left|B_{2}^{n}\right|^{1 / n} .
$$

Choosing $t=(\gamma m)^{1 / \alpha}$ we get the result.

We also need to recall the precise probabilistic form of the low $M^{*}$-estimate (which can be found in [17] and [27]): If $A$ is a symmetric convex body in $\mathbb{R}^{n}$ and if $\varepsilon, \delta \in(0,1)$, then we have

$$
R(A \cap F) \leq \frac{w(A)}{(1-\delta) \sqrt{\varepsilon}}
$$

for all $F$ in a subset $L_{n, k}$ of $G_{n, k}$ of measure $\nu_{n, k}\left(L_{n, k}\right) \geqslant 1-c_{1} \exp \left(-c_{2} \delta^{2} \varepsilon n\right)$, where $k=\lfloor(1-\varepsilon) n\rfloor$ and $c_{1}, c_{2}>0$ are absolute constants. Then, a well-known application of the low $M^{*}$-estimate (see e.g. [10, Theorem 2.1]) states that if $r>0$ is the solution of the equation

$$
\frac{w\left(A \cap r B_{2}^{n}\right)}{r}=\frac{1}{2} \sqrt{\varepsilon},
$$

then a typical $\lfloor(1-\varepsilon) n\rfloor$-dimensional central section of $A$ has radius smaller than $r$ (with probability greater than $1-\exp (-c \varepsilon n)$ ).

The main observation of this section, which essentially appears in [11, is the following: If $A$ is a symmetric convex body in $\mathbb{R}^{n}$ whose covering numbers $N\left(A, t B_{2}^{n}\right)$ are $\alpha$-regular for some $\alpha>0$, then one can get an upper bound for the diameter of random proportional sections of $A$.

Theorem 4.2. Let $\gamma \geqslant 1$, let $\alpha>0$ and let $A$ be a symmetric convex body in $\mathbb{R}^{n}$ which satisfies

$$
N\left(A, t B_{2}^{n}\right) \leqslant \exp \left(\frac{\gamma n}{t^{\alpha}}\right)
$$

for all $t \geqslant 1$. Then, for every $\varepsilon \in(0,1)$ a subspace $F \in G_{n,\lfloor(1-\varepsilon) n\rfloor \text { satisfies }}$

$$
R(A \cap F) \leqslant C \gamma^{\frac{1}{\alpha}} / \varepsilon^{\frac{1}{2}+\frac{1}{\alpha}},
$$


with probability greater than $1-c_{1} \exp \left(-c_{2} \varepsilon n\right)$, where $c_{1}, c_{2}, C>0$ are absolute constants.

Proof. Let $\varepsilon \in(0,1)$ and set $k=\lfloor(1-\varepsilon) n\rfloor$. We define $r>0$ by the equation

$$
w\left(A \cap r B_{2}^{n}\right)=\frac{1}{2} \sqrt{\varepsilon} r .
$$

From the precise probabilistic form of the low $M^{*}$-estimate (see above), we know that there exists a subset $L_{n, k}$ of $G_{n, k}$ with measure $\nu_{n, k}\left(L_{n, k}\right) \geqslant 1-c_{1} \exp \left(-c_{2} \varepsilon n\right)$ such that

$$
w(A \cap F) \leqslant R(A \cap F) \leqslant r
$$

for every $F \in L_{n, k}$. We use the following fact from [6]: if $X=\left(\mathbb{R}^{n},\|\cdot\|\right)$ is an $n$-dimensional normed space with unit ball $W$, and if $M=\int_{S^{n-1}}\|x\| d \sigma(x)$ and $b$ is the smallest positive constant for which $\|x\| \leqslant b\|x\|_{2}$ for all $x \in \mathbb{R}^{n}$, then there exist an integer $s \leqslant C(b / M)^{2}$ and $s$ orthogonal transformations $U_{1}, \ldots, U_{s} \in O(n)$ such that

$$
\frac{M}{2} B_{2}^{n} \subseteq \frac{1}{s} \sum_{i=1}^{s} U_{i}\left(W^{\circ}\right) \subseteq 2 M B_{2}^{n} .
$$

We apply this result for the body $W:=\left(A \cap r B_{2}^{n}\right)^{\circ}$. Note that $b=r$ and $M(W)=$ $w\left(A \cap r B_{2}^{n}\right)=\sqrt{\varepsilon} r / 2$, and hence we can find $s \leqslant \frac{c_{3}}{\varepsilon}$ and orthogonal transformations $U_{1}, \ldots, U_{s}$, satisfying

$$
\frac{1}{4} \sqrt{\varepsilon} r B_{2}^{n} \subseteq \frac{1}{s} \sum_{i=1}^{s} U_{i}\left(A \cap r B_{2}^{n}\right) \subseteq \sqrt{\varepsilon} r B_{2}^{n} .
$$

Set $A_{1}=\frac{1}{s} \sum U_{i}\left(A \cap r B_{2}^{n}\right)$. Now we can give an upper bound for $r$ using Lemma4.1. Clearly, the bodies $U_{i}\left(A \cap r B_{2}^{n}\right)$ satisfy

$$
N\left(U_{i}\left(A \cap r B_{2}^{n}\right), t B_{2}^{n}\right) \leqslant N\left(A, t B_{2}^{n}\right) \leqslant \exp \left(\frac{\gamma n}{t^{\alpha}}\right)
$$

for all $t \geqslant 1$; therefore

$$
\frac{1}{4} \sqrt{\varepsilon} r \leqslant\left(\frac{\left|A_{1}\right|}{\left|B_{2}^{n}\right|}\right)^{\frac{1}{n}} \leqslant c_{4} \gamma^{\frac{1}{\alpha}} s^{\frac{1}{\alpha}}
$$

This shows that

$$
R(A \cap F) \leqslant r \leqslant c_{5} \gamma^{\frac{1}{\alpha}} / \varepsilon^{\frac{1}{2}+\frac{1}{\alpha}}
$$

with probability greater than $1-c_{1} \exp \left(-c_{2} \varepsilon n\right)$.

\section{Projections of $L_{q}$-Centroid bodies}

Our aim is to obtain lower bounds for the inradius of proportional projections of $Z_{q}(\mu)$ and $Z_{q}^{\circ}(\mu)$. Let $1 \leqslant k \leqslant n-1$ and consider a random subspace $F \in G_{n, k}$. An upper bound for the radius of $Z_{q}(\mu) \cap F$, and hence a lower bound for the inradius of $P_{F}\left(Z_{q}^{\circ}(\mu)\right)$, follows from the low $M^{*}$-estimate (2.7) and (3.6).

Proposition 5.1. Let $\mu$ be an isotropic log-concave measure on $\mathbb{R}^{n}$. If $2 \leqslant q \leqslant$ $q_{*}(\mu)$ and if $\varepsilon \in(0,1)$ and $k=\lfloor(1-\varepsilon) n\rfloor$, then a subspace $F \in G_{n, k}$ satisfies

$$
R\left(Z_{q}(\mu) \cap F\right) \leqslant \frac{c_{1} \sqrt{q}}{\sqrt{\varepsilon}} \quad \text { or equivalently } \quad P_{F}\left(Z_{q}^{\circ}(\mu)\right) \supseteq \frac{c_{2} \sqrt{\varepsilon}}{\sqrt{q}} B_{F}
$$

with probability greater than $1-c_{3} \exp \left(-c_{4} \varepsilon n\right)$, where $c_{i}$ are absolute constants. 
We provide analogous upper bounds for $R\left(Z_{q}^{\circ}(\mu) \cap F\right), F \in G_{n, k}$. The idea of the proof comes from [21] (see the concluding remarks of this section). We start with the following immediate consequence of Theorem 4.2

Corollary 5.2. Let $A$ be a symmetric convex body in $\mathbb{R}^{m}$. Assume that $\gamma \geqslant 1$, $\alpha>0$, and $E$ is an ellipsoid in $\mathbb{R}^{m}$ such that

$$
N(A, t E) \leqslant \exp \left(\frac{\gamma m}{t^{\alpha}}\right)
$$

for all $t \geqslant 1$. Then, for every $\varepsilon \in(0,1)$ there exists $F \in G_{m,\lfloor(1-\varepsilon) m\rfloor}$ such that

$$
A \cap F \subseteq c \gamma^{\frac{1}{\alpha}} \varepsilon^{-\left(\frac{1}{2}+\frac{1}{\alpha}\right)} E \cap F,
$$

where $c>0$ is an absolute constant.

Proposition 5.3 (version for "small" $q$ ). Let $\mu$ be an isotropic log-concave measure on $\mathbb{R}^{n}$. Let $1 \leqslant \alpha<2$. For every $0<\varepsilon<1$ and any $q \leqslant \sqrt{\varepsilon n}$ there exist $k \geqslant(1-\varepsilon) n$ and $F \in G_{n, k}$ such that

$$
P_{F}\left(Z_{q}(\mu)\right) \supseteq c(2-\alpha) \varepsilon^{\frac{1}{2}+\frac{2}{\alpha}} \sqrt{q} B_{F} .
$$

Proof. Recall from (3.3) that for every $1 \leqslant m \leqslant n$ and any $H \in G_{n, m}$ we have

$$
P_{H}\left(Z_{q}(\mu)\right)=Z_{q}\left(\pi_{H}(\mu)\right) \text {. }
$$

In Section 3 we saw that if $\nu$ is an isotropic log-concave measure on $\mathbb{R}^{m}$, then

$$
\left|Z_{q}(\nu)\right|^{1 / m} \geqslant c \sqrt{q / m}
$$

for all $q \leqslant q_{-c}^{H}\left(\nu, \delta_{0}\right)$, where $\delta_{0} \geqslant 1$ is an absolute constant sufficiently large so that $q_{-c}^{H}\left(\nu, \delta_{0}\right) \geqslant c \sqrt{m}$. It follows that

$$
\left|P_{H}\left(Z_{q}(\mu)\right)\right|^{1 / m} \geqslant c_{1} \sqrt{q / m}
$$

for all $H \in G_{n, m}$ and all $q \leqslant \sqrt{m}$. We fix $1 \leqslant \alpha<2$ and consider an $\alpha$-regular $M$-ellipsoid $E$ of $Z_{q}(\mu)$, namely an ellipsoid such that

$$
\max \left\{N\left(Z_{q}(\mu), t E\right), N\left(E, t Z_{q}(\mu)\right)\right\} \leqslant e^{c(\alpha) n / t^{\alpha}}
$$

for all $t \geqslant 1$, where $c(\alpha) \leqslant C(2-\alpha)^{-\alpha / 2}$.

Let $0<\lambda_{1} \leqslant \cdots \leqslant \lambda_{n}$ be the axes of $E$, and let $\left\{u_{1}, \ldots, u_{n}\right\}$ be an orthonormal basis which corresponds to the $\lambda_{j}$. For every $1 \leqslant m, s \leqslant n$ we set

$$
H_{m}:=\operatorname{span}\left\{u_{1}, \ldots, u_{m}\right\} \quad \text { and } \quad F_{s}=\operatorname{span}\left\{u_{s+1}, \ldots, u_{n}\right\} .
$$

Since $E \cap H_{m}=P_{H_{m}}(E)$, we have

$$
N\left(P_{H_{m}}\left(Z_{q}(\mu)\right), t\left(E \cap H_{m}\right)\right) \leqslant N\left(Z_{q}(\mu), t E\right) \leqslant e^{c(\alpha) n / t^{\alpha}},
$$

and hence

$$
\left|P_{H_{m}}\left(Z_{q}(\mu)\right)\right|^{1 / m} \leqslant e^{c(\alpha) n /\left(t^{\alpha} m\right)}\left|B_{H_{m}}\right|^{1 / m}\left(t \lambda_{m}\right) .
$$

Choose $t=(c(\alpha) n / m)^{1 / \alpha}$. Assuming that $q \leqslant \sqrt{m}$, from (5.2) and (5.3) we get

$$
\lambda_{m} \geqslant\left(\frac{m}{c(\alpha) n}\right)^{1 / \alpha} \sqrt{q} \text {. }
$$


Next, let $0<\varepsilon<1$ and set $s=\left\lfloor\frac{\varepsilon n}{2}\right\rfloor$. We have

$$
N\left(E \cap F_{s}, t P_{F_{s}}\left(Z_{q}(\mu)\right)\right) \leqslant N\left(E, t Z_{q}(\mu)\right) \leqslant e^{c(\alpha) n / t^{\alpha}} \leqslant e^{2 c(\alpha)(n-s) / t^{\alpha}},
$$

for every $t \geqslant 1$.

We now use the duality of entropy theorem of Artstein-Avidan, Milman and Szarek [1]: There exist two absolute constants $a$ and $b>0$ such that for any dimension $n$ and any symmetric convex body $A$ in $\mathbb{R}^{n}$ one has

$$
N\left(B_{2}^{n}, a^{-1} A^{\circ}\right)^{1 / b} \leqslant N\left(A, B_{2}^{n}\right) \leqslant N\left(B_{2}^{n}, a A^{\circ}\right)^{b} .
$$

It follows that

$$
N\left(Z_{q}^{\circ}(\mu) \cap F_{s}, t E^{\circ} \cap F_{s}\right) \leqslant N\left(E \cap F_{s}, a t P_{F_{s}}\left(Z_{q}(\mu)\right)\right)^{b} \leqslant e^{c_{1}(\alpha)(n-s) / t^{\alpha}} .
$$

We apply Corollary 5.2 with the body $Z_{q}^{\circ}(\mu) \cap F_{s}$ (and $\gamma=c_{1}(\alpha)$ ) to find a subspace $F$ of $F_{s}$, of dimension $k \geqslant(1-\varepsilon / 2)(n-s) \geqslant(1-\varepsilon) n$, such that

$$
Z_{q}^{\circ}(\mu) \cap F \subseteq \frac{C}{\sqrt{2-\alpha} \varepsilon^{\frac{1}{2}+\frac{1}{\alpha}}} E^{\circ} \cap F
$$

and hence

$$
P_{F}\left(Z_{q}(\mu)\right) \supseteq c \sqrt{2-\alpha} \varepsilon^{\frac{1}{2}+\frac{1}{\alpha}} P_{F}(E) .
$$

From (5.4) we have

$$
E \cap F_{s} \supseteq \lambda_{s+1} B_{F_{s}} \supseteq c \sqrt{2-\alpha} \varepsilon^{1 / \alpha} \sqrt{q} B_{F_{s}},
$$

provided that $q \leqslant \sqrt{\varepsilon n}$. Then,

$$
\begin{aligned}
P_{F}(E) & =P_{F}\left(P_{F_{s}}(E)\right)=P_{F}\left(E \cap F_{s}\right) \supseteq c \sqrt{2-\alpha} \varepsilon^{1 / \alpha} \sqrt{q} P_{F}\left(B_{F_{s}}\right) \\
& =c \sqrt{2-\alpha} \varepsilon^{1 / \alpha} \sqrt{q} B_{F} .
\end{aligned}
$$

Combining this fact with (5.5) we conclude the proof.

Proposition 5.4 (version for "large" $q$ ). Let $\mu$ be an isotropic log-concave measure on $\mathbb{R}^{n}$. Let $1 \leqslant \alpha<2$. For every $0<\varepsilon<1$ and any $2 \leqslant q \leqslant \varepsilon n$ there exist $k \geqslant(1-\varepsilon) n$ and $F \in G_{n, k}$ such that

$$
P_{F}\left(Z_{q}(\mu)\right) \supseteq \frac{c_{1}(2-\alpha) \varepsilon^{\frac{1}{2}+\frac{2}{\alpha}}}{L_{\varepsilon n}} \sqrt{q} B_{F} \supseteq \frac{c_{2}(2-\alpha) \varepsilon^{\frac{1}{4}+\frac{2}{\alpha}}}{\sqrt[4]{n}} \sqrt{q} B_{F} .
$$

Proof. We apply the same argument more or less, only instead of the lower bounds (3.17), (3.18) for the volume radius of the $L_{q}$-centroid bodies we use (3.15). It follows that

$$
\left|P_{H}\left(Z_{q}(\mu)\right)\right|^{1 / m} \geqslant \frac{c_{1}}{L_{m}} \sqrt{q / m}
$$

for all $H \in G_{n, m}$ and all $q \leqslant m$. We define $H_{m}, F_{s}$ as in the proof of Proposition 5.3 and we consider an $\alpha$-regular $M$-ellipsoid $E$ of $Z_{q}(\mu)$. This time, assuming that $q \leqslant m$, from (5.6) and (5.3) we get

$$
\lambda_{m} \geqslant \frac{1}{L_{m}}\left(\frac{m}{c(\alpha) n}\right)^{1 / \alpha} \sqrt{q} .
$$

Next, fix some $\varepsilon \in(0,1)$, set $s=\left\lfloor\frac{\varepsilon n}{2}\right\rfloor$ and consider any $q \leqslant \varepsilon n / 2$. As previously, we find a subspace $F$ of $F_{s}$, of dimension $k \geqslant(1-\varepsilon / 2)(n-s) \geqslant(1-\varepsilon) n$, such that

$$
P_{F}\left(Z_{q}(\mu)\right) \supseteq c \sqrt{2-\alpha} \varepsilon^{\frac{1}{2}+\frac{1}{\alpha}} P_{F}(E)
$$


and

$$
P_{F}(E)=P_{F}\left(P_{F_{s}}(E)\right) \supseteq P_{F}\left(\lambda_{s+1} B_{F_{s}}\right) \supseteq \frac{c \sqrt{2-\alpha}}{L_{s}} \varepsilon^{1 / \alpha} \sqrt{q} B_{F} .
$$

Since $s \simeq \varepsilon n$ and $L_{\varepsilon n} \leqslant C \sqrt[4]{\varepsilon n}$, the result follows.

As we saw in Section 3 , if $K$ is an isotropic symmetric convex body in $\mathbb{R}^{n}$, then

$$
P_{F}(K) \supseteq P_{F}\left(Z_{q}(K)\right)
$$

for all $q>0$. Recall also that the measure $\mu_{K}$ with density $L_{K}^{n} \mathbf{1}_{K / L_{K}}$ is isotropic and $Z_{q}(K)=L_{K} Z_{q}\left(\mu_{K}\right)$. Choosing $q=\varepsilon n$ and applying Proposition 5.4 with $\mu=\mu_{K}$ we get:

Corollary 5.5. Let $K$ be a symmetric isotropic convex body in $\mathbb{R}^{n}$. For every $1 \leqslant \alpha<2$ and $0<\varepsilon<1$ there exist $k \geqslant(1-\varepsilon) n$ and $F \in G_{n, k}$ such that

$$
P_{F}(K) \supseteq L_{K} P_{F}\left(Z_{q}\left(\mu_{K}\right)\right) \supseteq c(2-\alpha) \varepsilon^{\frac{3}{4}+\frac{2}{\alpha}} \sqrt[4]{n} L_{K} B_{F} .
$$

Remark 5.6. Some variants of Corollary 5.5 have appeared in the literature before. In [35] a stronger estimate is obtained with a different method: If $K$ is a symmetric isotropic convex body in $\mathbb{R}^{n}$, then for any $0<\varepsilon<1$, there exists a subspace $F$ of $\mathbb{R}^{n}$ with $\operatorname{dim} F \geqslant(1-\varepsilon) n$ such that

$$
P_{F}(K) \supseteq c \varepsilon^{3 / 2} \frac{\sqrt[4]{n}}{\log n} L_{K} B_{F}
$$

where $c>0$ is an absolute constant. A similar result with cubic dependence on $\varepsilon$ appears in 3]. Our argument is very much related to the one in [21, where, under the additional assumption that $L_{n} \leqslant C$ for all $n \geqslant 1$, the existence of some $F \in G_{n,\lfloor(1-\varepsilon) n\rfloor}$ so that

$$
P_{F}(K) \supseteq c \varepsilon^{3} \sqrt{n} B_{F}
$$

is established for all isotropic convex bodies $K$ in $\mathbb{R}^{n}$ and all $0<\varepsilon<1$. Under this assumption, our argument would result in the estimate $P_{F}(K) \supseteq c(2-\alpha) \varepsilon^{\frac{\alpha+2}{\alpha}} \sqrt{n} B_{F}$ for all $1 \leqslant \alpha<2$.

Remark 5.7. Proposition 5.3 and Proposition 5.4 guarantee the existence of one $\lfloor(1-\varepsilon) n\rfloor$-dimensional projection of $Z_{q}(\mu)$ with "large" inradius. However, it is proved in [13] that for every fixed proportion $\mu \in(0,1)$ and every $0<s<1 /(2-\mu)$, the maximal inradius of $\lfloor\mu n\rfloor$-dimensional projections and the random inradius of $\lfloor s \mu n\rfloor$-dimensional projections of a symmetric convex body $K$ in $\mathbb{R}^{n}$ are comparable up to a constant depending on $\mu$ and $s$. More precisely, if $a(\lambda, K)$ denotes the maximal (and if $b(\lambda, K)$ denotes the "random") inradius of a $\lfloor\lambda n\rfloor$-dimensional projection of $K$, then

$$
\left(\frac{c \mu(1-s(2-\mu))}{1-s \mu} \sqrt{1-\mu}\right) a(\mu, K) \leqslant b(s \mu, K)
$$

for every $n \geqslant n_{0}(\mu, s)$. Using this fact one can obtain versions of the results of this section concerning random proportional projections of $Z_{q}(\mu)$. Since an estimate for the maximal inradius is sufficient for our subsequent work in this paper, we do not present the precise statements. 


\section{Covering Numbers}

Using Proposition 5.3, for any isotropic log-concave measure $\mu$ on $\mathbb{R}^{n}$ we can get some estimates for the covering numbers $N\left(\sqrt{q} B_{2}^{n}, t Z_{q}(\mu)\right)$. These will follow from an entropy extension result from [24]:

Lemma 6.1. Let $K, L$ be symmetric convex bodies in $\mathbb{R}^{n}$ and assume that $L \subseteq R K$. Let $F$ be a subspace of $\mathbb{R}^{n}$ with $\operatorname{dim} F=n-m$ and let $0<r<t<R$. Then, we have

$$
N(L, t K) \leqslant 2^{m}\left(\frac{2 R+t}{t-r}\right)^{m} N\left(P_{F}(L), \frac{r}{2} P_{F}(K)\right) .
$$

Remark 6.2. Alternatively, one might use an analogous result, due to Vershynin and Rudelson (see [36, Lemma 5.2]): If $K$ is a symmetric convex body in $\mathbb{R}^{n}$ such that $K \supseteq \delta B_{n}$ and if $P_{F}(K) \supseteq B_{F}$ for some $F \in G_{n, k}, k \geqslant(1-\varepsilon) n$, then

$$
N\left(B_{2}^{n}, 4 K\right) \leqslant(C / \delta)^{2 \varepsilon n} .
$$

The reader may wish to check that applying this fact instead of Lemma 6.1 leads to the same estimate in Proposition 6.3 below.

Proposition 6.3. Let $\mu$ be an isotropic log-concave measure on $\mathbb{R}^{n}$. Assume that $q \leqslant \sqrt{n}$. Then, for any $1 \leqslant \alpha<2$ and any

$$
1 \leqslant t \leqslant \min \left\{\sqrt{q}, c_{2}(2-\alpha)^{-1}\left(n / q^{2}\right)^{\frac{\alpha+4}{2 \alpha}}\right\}
$$

we have

$$
\max \left\{\log N\left(\sqrt{q} B_{2}^{n}, t Z_{q}(\mu)\right), \log N\left(\sqrt{q} Z_{q}^{\circ}(\mu), t B_{2}^{n}\right)\right\}
$$

$$
\leqslant c(\alpha) \frac{n}{t^{\frac{2 \alpha}{\alpha+4}}} \max \left\{\log \frac{\sqrt{2 q}}{t}, \log \frac{1}{(2-\alpha) t}\right\},
$$

where $c(\alpha) \leqslant C(2-\alpha)^{-2 / 3}$.

Proof. Note that since $B_{2}^{n} \subseteq Z_{q}(\mu)$, the interesting range for $t$ is up to $\sqrt{q}$. Given some $\varepsilon \in(0,1)$, let $k=(1-\varepsilon) n$ and $F \in G_{n, k}$. Applying Lemma 6.1 for the bodies $\sqrt{q} B_{2}^{n}$ and $Z_{q}(\mu)$ with $R=\sqrt{q}$ and $r=t / 2$ we see that for every $1 \leqslant t<\sqrt{q}$,

$$
N\left(\sqrt{q} B_{2}^{n}, t Z_{q}(\mu)\right) \leqslant\left(\frac{c_{1} \sqrt{q}}{t}\right)^{\varepsilon n} N\left(\sqrt{q} B_{F}, \frac{t}{4} P_{F}\left(Z_{q}(\mu)\right)\right) .
$$

If $q^{2} \leqslant \varepsilon n$, then Proposition 5.3 shows that for every $1 \leqslant \alpha<2$, there exists $F \in G_{n, k}, k=(1-\varepsilon) n$, such that $P_{F}\left(Z_{q}(\mu)\right) \supseteq c_{2}(2-\alpha) \varepsilon^{\frac{1}{2}+\frac{2}{\alpha}} \sqrt{q} B_{F}$. Thus, we arrive at

$$
N\left(\sqrt{q} B_{2}^{n}, t Z_{q}(\mu)\right) \leqslant\left(\frac{c_{3} \sqrt{q}}{t}\right)^{\varepsilon n} N\left(B_{F}, c_{4} t(2-\alpha) \varepsilon^{\frac{1}{2}+\frac{2}{\alpha}} B_{F}\right) .
$$

In the end we choose $\varepsilon \simeq[(2-\alpha) t]^{-\frac{2 \alpha}{\alpha+4}}$ (the restriction $[(2-\alpha) t]^{\frac{2 \alpha}{\alpha+4}} \leqslant \mathrm{cn} / \mathrm{q}^{2}$ is needed at this point-note that if e.g. $q \leqslant n^{3 / 7}$, then this allows us to consider any $t$ up to $\sqrt{q}$ ). With this choice of $\varepsilon$, we get from (6.4) that

$$
\log N\left(\sqrt{q} B_{2}^{n}, t Z_{q}(\mu)\right) \leqslant c(\alpha) \frac{n \log \left(2 q / t^{2}\right)}{t^{\frac{2 \alpha}{\alpha+4}}} .
$$

This proves the upper bound for the first covering number in (6.2) provided that $t \geqslant c_{1}(2-\alpha)^{-1}\left(\right.$ since $(2-\alpha) t \simeq \varepsilon^{\frac{\alpha+4}{2 \alpha}}$ must be less than 1$)$. 
When $1 \leq t \leq c(2-\alpha)^{-1}$ we use the inequality

$$
N\left(\sqrt{q} B_{2}^{n}, t Z_{q}(\mu)\right) \leqslant N\left(\sqrt{q} B_{2}^{n}, c_{1}(2-\alpha)^{-1} Z_{q}(\mu)\right) N\left(Z_{q}(\mu), c_{1}^{-1}(2-\alpha) t Z_{q}(\mu)\right) .
$$

Noticing that the latter covering number is less than $\left(1+c(2-\alpha)^{-1} t^{-1}\right)^{n}$ completes the proof for the first covering number in (6.2) after elementary calculations.

The bound for the second covering number in (6.2) follows from the duality of entropy theorem.

Using Proposition 5.4 instead of Proposition 5.3 we get the following:

Proposition 6.4. Let $\mu$ be an isotropic log-concave measure on $\mathbb{R}^{n}$. Assume that $2 \leqslant q \leqslant n$. Then, for any $1 \leqslant \alpha<2$ and any

$$
1 \leqslant t \leqslant \min \left\{\sqrt{q}, c_{2}(2-\alpha)^{-1} L_{n}\left(\frac{n}{q}\right)^{\frac{\alpha+4}{2 \alpha}}\right\}
$$

we have

$$
\begin{aligned}
& \max \left\{\log N\left(\sqrt{q} B_{2}^{n}, t Z_{q}(\mu)\right), \log N\left(\sqrt{q} Z_{q}^{\circ}(\mu), t B_{2}^{n}\right)\right\} \\
& \leqslant c(\alpha) L_{n}^{\frac{2 \alpha}{\alpha+4}} \frac{n}{t^{\frac{2 \alpha}{\alpha+4}}} \max \left\{\log \frac{\sqrt{2 q}}{t}, \log \frac{L_{n}}{(2-\alpha) t}\right\},
\end{aligned}
$$

where $c(\alpha) \leqslant C(2-\alpha)^{-2 / 3}$ and $c_{1}, c_{2}, C>0$ are absolute constants.

Proof. We proceed along the same lines as before, and the only other thing that we need to take into account is the fact that for every $\varepsilon \in(0,1), L_{\varepsilon n} \leqslant c L_{n}$ for some absolute constant $c$. Note that here we are allowed to consider any $t$ up to $\sqrt{q}$ if we restrict ourselves to those $q$ that do not exceed $\sqrt{L_{n}} n^{3 / 4}$.

Remark 6.5. If we do not use the monotonicity of $L_{n}$, but rather we use the bound $L_{\varepsilon n} \leqslant \sqrt[4]{\varepsilon n}$, we will end up with an upper bound of the form

$$
\frac{C}{(2-\alpha)^{\frac{4 \alpha}{\alpha+8}}} \frac{n^{\frac{2 \alpha+8}{\alpha+8}} \log q}{t^{\frac{4 \alpha}{\alpha+8}}}
$$

for $\max \left\{\log N\left(\sqrt{q} B_{2}^{n}, t Z_{q}(\mu)\right), \log N\left(\sqrt{q} Z_{q}^{\circ}(\mu), t B_{2}^{n}\right)\right\}$. We thus get a better exponent of $t$ for each $\alpha$, but the restrictions in the proof force $t$ to be in the range

$$
c(2-\alpha)^{-1} \sqrt[4]{n} \leqslant t \leqslant c(2-\alpha)^{-1} \frac{n^{\frac{2 \alpha+8}{4 \alpha}}}{q^{\frac{\alpha+8}{4 \alpha}}}
$$

(again $t$ can vary up to $\sqrt{q}$ if, for example, $q \leqslant n^{6 / 7}$ ). In this range, the latter bound for the covering numbers is more efficient only if $L_{n}$ depends "badly" on $n$.

\section{UPPER BOUND FOR $M\left(Z_{q}(\mu)\right)$}

To make use of the covering estimates we have just obtained so as to give an upper bound for $M\left(Z_{q}(\mu)\right.$ ), we employ the Dudley-Fernique decomposition (see e.g. [9, §2.5.2]). We consider the symmetric convex body $K:=\sqrt{q} Z_{q}^{\circ}(\mu)$ and, for any $1 \leqslant j \leqslant \log q$, we consider the entropy number $N\left(K, 2^{-j} R B_{2}^{n}\right)$, where $R=R(K) \leqslant \sqrt{q}$. There exists $N_{j} \subseteq K$ with $\left|N_{j}\right|=N\left(K, 2^{-j} R B_{2}^{n}\right)$ such that for 
any $x \in K$, there exists $v \in N_{j}$ satisfying $\|x-v\|_{2} \leqslant 2^{-j} R$. We set $N_{0}=\{0\}$ and $Z_{j}=N_{j}-N_{j-1}$. Then, we have:

Lemma 7.1. Let $K$ be a symmetric convex body in $\mathbb{R}^{n}$. For any $m \in \mathbb{N}$ and any $x \in K$ there exist $z_{1}, \ldots, z_{m}, w_{m}$ with $z_{j} \in Z_{j} \cap \frac{3 R}{2^{j}} B_{2}^{n}$ and $w_{m} \in \frac{R}{2^{m}} B_{2}^{n}$ such that

$$
x=z_{1}+\cdots+z_{m}+w_{m},
$$

where $R=R(K)$ is the radius of $K$.

Theorem 7.2 (version for small $q$ ). Let $\mu$ be an isotropic log-concave measure on $\mathbb{R}^{n}$. For every $1 \leqslant \alpha<2$, for every

$$
1 \leqslant q \leqslant c(2-\alpha)^{-1 / 6} n^{\frac{\alpha+4}{3 \alpha+8}}
$$

we have

$$
M\left(Z_{q}(\mu)\right) \leqslant C(2-\alpha)^{-1 / 3} \frac{\sqrt{\max \left\{\log q, \log (2-\alpha)^{-1}\right\}}}{q^{\frac{\alpha}{2 \alpha+8}}},
$$

where $C>0$ is an absolute constant. In particular, for every $1 \leqslant q \leqslant n^{3 / 7}$,

$$
M\left(Z_{q}(\mu)\right) \leqslant C \frac{(\log q)^{5 / 6}}{\sqrt[6]{q}} .
$$

Proof. We set $K=\sqrt{q} Z_{q}^{\circ}(\mu)$. Using Lemma 7.1, for any $m \in \mathbb{N}$ and any $x \in K$ we can find $\left(z_{j}\right)_{j \leqslant m} \subset Z_{j} \cap \frac{3 R}{2^{j}} B_{2}^{n}$ and $w_{m} \in \frac{R}{2^{m}} B_{2}^{n}$ such that $x=z_{1}+\cdots+z_{m}+w_{m}$. For any $\theta \in S^{n-1}$ one has

$$
|\langle x, \theta\rangle| \leqslant \sum_{j=1}^{m}\left|\left\langle z_{j}, \theta\right\rangle\right|+\left|\left\langle w_{m}, \theta\right\rangle\right| .
$$

We write $\bar{z}=z /\|z\|_{2}$ for all $z \neq 0$. We have

$$
\begin{aligned}
w(K) & =\int_{S^{n-1}} \max _{x \in K}|\langle x, \theta\rangle| d \sigma(\theta) \\
& \leqslant \sum_{j=1}^{m} \int_{S^{n-1}} \max _{z \in Z_{j}}|\langle\theta, z\rangle| d \sigma(\theta)+\int_{S^{n-1}} \max _{w \in 2^{-m} R B_{2}^{n}}|\langle w, \theta\rangle| d \sigma(\theta) \\
& \leqslant \sum_{j=1}^{m} \frac{3 R}{2^{j}} \int_{S^{n-1}} \max _{z \in Z_{j}}|\langle\theta, \bar{z}\rangle| d \sigma(\theta)+\frac{R}{2^{m}} \\
& \leqslant \sum_{j=1}^{m} \frac{c_{3} R}{2^{j}} \frac{\sqrt{\log \left|Z_{j}\right|}}{\sqrt{n}}+\frac{R}{2^{m}},
\end{aligned}
$$

where we have used the following:

Fact. For any $u_{1}, \ldots, u_{N} \in S^{n-1}$ we have

$$
\int_{S^{n-1}} \max _{j \leqslant N}\left|\left\langle\theta, u_{j}\right\rangle\right| d \sigma(\theta) \leqslant c_{3} \frac{\sqrt{\log N}}{\sqrt{n}} .
$$

By the definition of $Z_{j}$ and Proposition 6.3 we obtain

(7.8) $\log \left|Z_{j}\right| \leqslant \log \left|N_{j}\right|+\log \left|N_{j-1}\right| \leqslant c(\alpha) n\left(\frac{2^{j}}{R}\right)^{\frac{2 \alpha}{\alpha+4}} \max \left\{\log q, \log (2-\alpha)^{-1}\right\}$, 
where we assume that $R / 2^{m} \geqslant 1$ and $c(\alpha) \leqslant C(2-\alpha)^{-\frac{2 \alpha}{\alpha+4}}$. Plugging this into (17.6) we conclude that

$$
\begin{aligned}
w(K) & \leqslant \frac{c_{5}}{(2-\alpha)^{\frac{\alpha}{\alpha+4}}} R^{\frac{4}{\alpha+4}} \sqrt{\max \left\{\log q, \log \frac{1}{2-\alpha}\right\}} \sum_{j \leqslant m} \frac{1}{2^{\frac{4 j}{\alpha+4}}}+\frac{R}{2^{m}} \\
& \leqslant \frac{C}{(2-\alpha)^{\frac{\alpha}{\alpha+4}}} q^{\frac{2}{\alpha+4}} \sqrt{\max \left\{\log q, \log \frac{1}{2-\alpha}\right\}}
\end{aligned}
$$

if $m$ is large enough so that $R / 2^{m} \simeq 1$. It remains to observe that

$$
w(K)=\sqrt{q} w\left(Z_{q}^{\circ}(\mu)\right)=\sqrt{q} M\left(Z_{q}(\mu)\right),
$$

and hence

$$
M\left(Z_{q}(\mu)\right) \leqslant \frac{C}{(2-\alpha)^{\frac{\alpha}{\alpha+4}}} \frac{\sqrt{\max \left\{\log q, \log (2-\alpha)^{-1}\right\}}}{q^{\frac{\alpha}{2 \alpha+8}}} .
$$

Finally, to obtain (7.4), we set $\alpha=2-\frac{1}{\log q}$.

Given a symmetric isotropic convex body $K$ in $\mathbb{R}^{n}$ we apply Theorem 7.2 with the isotropic measure $\mu_{K}$, that has density $L_{K}^{n} \mathbf{1}_{K / L_{K}}$, and with $q=n^{3 / 7}$ (which is the optimal choice for this purpose). Then we get:

Theorem 7.3. Let $K \subset \mathbb{R}^{n}$ be isotropic and symmetric. Then,

$$
M(K) \leqslant C \frac{(\log n)^{5 / 6}}{L_{K} \sqrt[14]{n}} .
$$

Using Proposition 6.4 instead of Proposition 6.3, we also get:

Theorem 7.4. Let $\mu$ be an isotropic log-concave measure on $\mathbb{R}^{n}$. For every $1 \leqslant$ $\alpha<2$, for every

$$
1 \leqslant q \leqslant c_{1}(2-\alpha)^{-1 / 3} L_{n}^{\frac{2 \alpha}{2 \alpha+4}} n^{\frac{\alpha+4}{2 \alpha+4}},
$$

we have

$$
M\left(Z_{q}(\mu)\right) \leqslant C(2-\alpha)^{-1 / 3} L_{n}^{\frac{\alpha}{\alpha+4}} \frac{\sqrt{\max \left\{\log q, \log L_{n}(2-\alpha)^{-1}\right\}}}{q^{\frac{\alpha}{2 \alpha+8}}},
$$

where $c_{1}, C>0$ are absolute constants. In particular, for every $q$ such that $L_{n} \log q \leqslant q \leqslant \sqrt{L_{n}} n^{3 / 4}$,

$$
M\left(Z_{q}(\mu)\right) \leqslant C \frac{\sqrt[3]{L_{n}}(\log q)^{5 / 6}}{\sqrt[6]{q}}
$$

and, for every symmetric isotropic convex body $K$ in $\mathbb{R}^{n}$,

$$
M(K) \leqslant C \frac{\sqrt[4]{L_{n}}(\log n)^{5 / 6}}{L_{K} \sqrt[8]{n}}
$$

Remark 7.5. Similarly, using Remark 6.5 instead of Proposition 6.3, we see that for every $1 \leqslant \alpha<2$, for every

$$
c_{1}(2-\alpha)^{-2} \sqrt{n} \leqslant q \leqslant c_{2}(2-\alpha)^{-1 / 3} n^{\frac{2 \alpha+8}{3 \alpha+8}},
$$


we have

$$
M\left(Z_{q}(\mu)\right) \leqslant C(2-\alpha)^{-2 / 5} \sqrt[4]{n^{\frac{2 \alpha}{\alpha+8}}} \frac{\sqrt{\log q}}{q^{\frac{\alpha}{\alpha+8}}},
$$

where $c_{1}, c_{2}$ and $C>0$ are absolute constants, and for every $\sqrt{n} \log n \leqslant q \leqslant n^{6 / 7}$,

$$
M\left(Z_{q}(\mu)\right) \leqslant C \frac{\sqrt[10]{n}(\log n)^{9 / 10}}{\sqrt[5]{q}} .
$$

Therefore, for every isotropic symmetric convex body $K$ in $\mathbb{R}^{n}$,

$$
M(K) \leqslant C \frac{(\log n)^{9 / 10}}{L_{K} \sqrt[14]{n}} .
$$

\section{Further OBSERVATIONS}

In this last section we collect a number of additional observations on the geometry of the centroid bodies $Z_{q}(\mu)$.

1. Inradius of projections. We first provide lower bounds for $R\left(Z_{q}(\mu) \cap F\right)$ and $R\left(Z_{q}^{\circ}(\mu) \cap F\right)$; actually, they hold true for every $1 \leqslant k<n$ and any $F \in G_{n, k}$. By duality, these estimates (combined with e.g. Proposition 5.3) determine the inradius of $P_{F}\left(Z_{q}^{\circ}(\mu)\right)$ and $P_{F}\left(Z_{q}(\mu)\right)$. Our starting point is the next proposition, which can essentially be found in [11].

Proposition 8.1. Let $A$ be a symmetric convex body in $\mathbb{R}^{n}$. Assume that there exists $\gamma \geqslant 1$ such that

$$
N\left(B_{2}^{n}, t A\right) \leqslant \exp \left(\frac{\gamma n}{t^{p}}\right)
$$

for every $t \geqslant 1$. For every $\delta \in(0,1)$ and every $F \in G_{n,\lfloor\delta n\rfloor}$ we have

$$
w(A \cap F) \geqslant \frac{c \delta^{1 / p}}{\gamma^{1 / p}} .
$$

Proof. Let $k=\lfloor\delta n\rfloor$ and consider any $F \in G_{n, k}$. Using the assumption and the duality of entropy theorem we see that the projection $P_{F}\left(A^{\circ}\right)$ of $A^{\circ}$ onto $F$ satisfies

$$
N\left(P_{F}\left(A^{\circ}\right), t B_{F}\right) \leqslant N\left(A^{\circ}, t B_{2}^{n}\right) \leqslant \exp \left(\frac{\gamma k}{\delta t^{p}}\right),
$$

for every $t \geqslant 1$. We apply Theorem 4.2 with $W=P_{F}\left(A^{\circ}\right), n=k$ and $\varepsilon=1 / 2$. There exists $H \in G_{k,\lfloor k / 2\rfloor}(F)$ for which

$$
P_{F}\left(A^{\circ}\right) \cap H \subseteq \frac{c \gamma^{1 / p}}{\delta^{1 / p}} B_{H} .
$$

Taking polars in $H$ we see that $P_{H}(A \cap F) \supseteq \frac{c \delta^{1 / p}}{\gamma^{1 / p}} B_{H}$.

Recall now that, given a symmetric convex body $C$ in $\mathbb{R}^{m}$ and an $s$-dimensional subspace $L$ of $\mathbb{R}^{m}$, one has $M(C \cap L) \lesssim \sqrt{m / s} M(C)$ (see [12, Section 4.2]). Therefore, setting $C=(A \cap F)^{\circ}$ and $L=H$, we obtain

$$
\begin{aligned}
w(A \cap F) & =M\left((A \cap F)^{\circ}\right) \geqslant \frac{1}{\sqrt{2}} M\left((A \cap F)^{\circ} \cap H\right)=\frac{c}{\sqrt{2}} w\left(P_{H}(A \cap F)\right) \\
& \geqslant \frac{c^{\prime} \delta^{1 / p}}{\gamma^{1 / p}}
\end{aligned}
$$

as claimed. 
Theorem 8.2. Let $\mu$ be an isotropic log-concave measure on $\mathbb{R}^{n}$. Assume that $q \leqslant \sqrt{n}$. Then, for any $1 \leqslant \alpha<2,0<\delta<1$ and any $F \in G_{n,\lfloor\delta n\rfloor}$ we have

$$
R\left(Z_{q}(\mu) \cap F\right) \geqslant w\left(Z_{q}(\mu) \cap F\right) \geqslant c \frac{(2-\alpha) \delta^{\frac{\alpha+4}{2 \alpha}}}{\left(\log \frac{1}{2-\alpha}\right)^{\frac{\alpha+4}{2 \alpha}}} \sqrt{q} .
$$

Proof. From Proposition 6.3 we know that

$$
\log N\left(\sqrt{q} B_{2}^{n}, t Z_{q}(\mu)\right) \leqslant c(\alpha) \frac{n}{t^{\frac{2 \alpha}{\alpha+4}}} \max \left\{\log \frac{\sqrt{2 q}}{t}, \log \frac{1}{(2-\alpha) t}\right\},
$$

where $c(\alpha) \leqslant C(2-\alpha)^{-2 / 3}$. Thus, we may apply Proposition 8.1 with

$$
\gamma=c(\alpha) \frac{\max \left\{\log q, \log \frac{1}{2-\alpha}\right\}}{\sqrt{q}^{p}}
$$

and $p=\frac{2 \alpha}{\alpha+4}$.

A similar argument applies to $Z_{q}^{\circ}(\mu)$. Since $M\left(Z_{q}^{\circ}(\mu)\right)=w\left(Z_{q}(\mu)\right) \simeq \sqrt{q}$ for all $q \leqslant \sqrt{n}$, from the dual Sudakov inequality we have

$$
\log N\left(B_{2}^{n}, t \sqrt{q} Z_{q}^{\circ}(\mu)\right) \leqslant \frac{c n}{t^{2}}
$$

for all $t \geqslant 1$. Applying Proposition 8.1 with $\gamma=c q$ and $p=2$ we get:

Theorem 8.3. Let $\mu$ be an isotropic log-concave measure on $\mathbb{R}^{n}$. Assume that $q \leqslant \sqrt{n}$. Then, for any $1 \leqslant \alpha<2,0<\delta<1$ and any $F \in G_{n,\lfloor\delta n\rfloor}$ we have

$$
R\left(Z_{q}^{\circ}(\mu) \cap F\right) \geqslant w\left(Z_{q}^{\circ}(\mu) \cap F\right) \geqslant \frac{c \sqrt{\delta}}{\sqrt{q}} .
$$

2. Upper bound for $M_{-k}\left(Z_{q}(\mu)\right)$. Let $C$ be a symmetric convex body in $\mathbb{R}^{n}$. For every $p \neq 0$, one can define

$$
M_{p}(C):=\left(\int_{S^{n-1}}\|\theta\|_{C}^{p} d \sigma(\theta)\right)^{1 / p} .
$$

Litvak, Milman and Schechtman proved in 23 that if $b$ is the smallest constant for which $\|x\| \leqslant b\|x\|_{2}$ holds true for every $x \in \mathbb{R}^{n}$, then

$$
\max \left\{M(C), c_{1} \frac{b \sqrt{q}}{\sqrt{n}}\right\} \leqslant M_{p}(C) \leqslant \max \left\{2 M(C), c_{2} \frac{b \sqrt{q}}{\sqrt{n}}\right\}
$$

for all $p \in[1, n]$, where $c_{1}, c_{2}>0$ are absolute constants. In particular,

$$
M_{p}(C) \simeq M(C)
$$

as long as $p \leqslant k(C):=k_{*}\left(C^{\circ}\right)$. Klartag and Vershynin defined in 22 the parameter

$$
d(C)=\min \left\{-\log \sigma\left(\left\{x \in S^{n-1}:\|x\| \leqslant \frac{M(C)}{2}\right\}\right), n\right\},
$$

and they observed that $d(C)$ is always larger than $k(C)$. Their main result is an analogue of (8.2) for negative values of $p$ : one has

$$
M_{-p}(C) \simeq M(C)
$$

as long as $0<p \leqslant d(C)$.

Let $\mu$ be an isotropic log-concave measure on $\mathbb{R}^{n}$. Since $M_{-p}\left(Z_{q}(\mu)\right)$ is clearly smaller than $M\left(Z_{q}(\mu)\right)$, our next aim is to provide upper bounds for these quantities 
and to compare them to the ones from Section 7. We will use a formula for $M_{-k}(C)$ which appears in 31.

Lemma 8.4. Let $C$ be a symmetric convex body in $\mathbb{R}^{n}$. For every integer $1 \leqslant k<n$,

$$
M_{-k}(C) \simeq\left(\int_{G_{n, k}} \operatorname{vrad}\left(P_{F}\left(C^{\circ}\right)\right)^{-k} d \nu_{n, k}(F)\right)^{-1 / k} .
$$

The proof is simple: using the Blaschke-Santaló and the reverse Santaló inequality (see [5]) one can write

$$
\begin{aligned}
M_{-k}^{-1}(C) & =\left(\int_{S^{n-1}} \frac{1}{\|x\|_{C}^{k}} d \sigma(x)\right)^{1 / k} \\
& =\left(\frac{1}{\omega_{k}} \int_{G_{n, k}} \omega_{k} \int_{S_{F}} \frac{1}{\|x\|_{C \cap F}^{k}} d \sigma(x) d \nu_{n, k}(F)\right)^{1 / k} \\
& =\left(\int_{G_{n, k}} \frac{|C \cap F|}{\left|B_{2}^{k}\right|} d \nu_{n, k}(F)\right)^{1 / k} \\
& \simeq\left(\int_{G_{n, k}} \frac{\left|B_{2}^{k}\right|}{\left|P_{F}\left(C^{\circ}\right)\right|} d \nu_{n, k}(F)\right)^{1 / k} .
\end{aligned}
$$

Proposition 8.5. Let $\mu$ be an isotropic log-concave measure on $\mathbb{R}^{n}$. For every $2 \leqslant q \leqslant \sqrt{n}$ and $k \geqslant q^{2} \log ^{2} q$ one has

$$
M_{-k}\left(Z_{q}(\mu)\right) \leqslant \frac{c \log ^{3} q}{\sqrt{q}}\left(\frac{n}{k}\right)^{3 / 2} .
$$

Proof. We choose $\alpha=2-\frac{1}{\log q}$. From the proof of Proposition 6.3 we see that if $t \leq \sqrt{q}$ and

$$
\frac{q^{2}}{(\log q)^{2 / 3}} t^{2 / 3} \leqslant n
$$

then

$$
N\left(\sqrt{q} Z_{q}^{\circ}(\mu), t B_{2}^{n}\right) \leqslant \exp \left(c_{1} \frac{n(\log q)^{2 / 3}}{t^{2 / 3}} \log \frac{2 q}{t^{2}}\right)
$$

where $c_{1}$ is an absolute constant. Thus, for any integer $1 \leqslant k<n$ and any $F \in G_{n, k}$ we have

$$
\begin{aligned}
\sqrt{q}\left|P_{F}\left(Z_{q}^{\circ}(\mu)\right)\right|^{1 / k} & \leqslant t\left|B_{2}^{k}\right|^{1 / k} N\left(\sqrt{q} P_{F}\left(Z_{q}^{\circ}(\mu)\right), t B_{F}\right)^{1 / k} \\
& \leqslant t\left|B_{2}^{k}\right|^{1 / k} \exp \left(c_{1} \frac{n(\log q)^{2 / 3}}{k t^{2 / 3}} \log \frac{2 q}{t^{2}}\right) .
\end{aligned}
$$

Choosing $t \simeq(n / k)^{3 / 2} \log ^{3} q$ we conclude that

$$
\left(\frac{\left|P_{F}\left(Z_{q}^{\circ}(\mu)\right)\right|}{\left|B_{2}^{k}\right|}\right)^{1 / k} \leqslant \frac{c \log ^{3} q}{\sqrt{q}}\left(\frac{n}{k}\right)^{3 / 2},
$$

and the result follows from Lemma 8.4 
Note. Since $Z_{q}(\mu) \supseteq B_{2}^{n}$, one has the obvious upper bound $M_{-k}\left(Z_{q}(\mu)\right) \leqslant 1$ for all $1 \leqslant k<n$. So, the estimate of Proposition 8.5 is non-trivial provided that $k \geqslant n \log ^{2} q / q^{1 / 3}$.

Remark 8.6. Similarly, starting from Proposition 6.4 and following the proof of Proposition 8.5, we obtain the following.

Proposition 8.7. Let $\mu$ be an isotropic log-concave measure on $\mathbb{R}^{n}$. For every $2 \leqslant q \leqslant n$ and $k \geqslant L_{n}^{2 / 3} q$ one has

$$
M_{-k}\left(Z_{q}(\mu)\right) \leqslant \frac{c L_{n}}{\sqrt{q}}\left(\frac{n}{k}\right)^{3 / 2}
$$

In view of (8.3) a natural question is to give lower bounds for $d\left(Z_{q}(\mu)\right)$, because if $d\left(Z_{q}(\mu)\right)$ is large enough so that we can use Proposition 8.5 or Proposition 8.7, then we would have an alternative source of possibly better information on $M\left(Z_{q}(\mu)\right)$ as well. What we know is a simple lower bound for $k\left(Z_{q}(\mu)\right)$ and $k\left(Z_{q}^{\circ}(\mu)\right)$ in the range $2 \leqslant q \leqslant q_{*}(\mu)$. For every $2 \leqslant q \leqslant q_{*}(\mu)$ one has

$$
\min \left\{k\left(Z_{q}(\mu)\right), k\left(Z_{q}^{\circ}(\mu)\right)\right\} \geqslant \frac{c_{1} n}{q},
$$

where $c_{1}$ is an absolute constant.

To see this, recall that $B_{2}^{n} \subseteq Z_{q}(\mu) \subseteq c_{2} q B_{2}^{n}$, and hence

$$
R\left(Z_{q}(\mu)\right) \leqslant c_{2} q \quad \text { and } \quad R\left(Z_{q}^{\circ}(\mu)\right) \leqslant 1 .
$$

Then, using (3.6) we get

$$
k_{*}\left(Z_{q}(\mu)\right) \geqslant c_{3} n \frac{w^{2}\left(Z_{q}(\mu)\right)}{R^{2}\left(Z_{q}(\mu)\right)} \geqslant c_{4} n \frac{q}{q^{2}}=\frac{c_{4} n}{q} .
$$

Also, using the fact that $w\left(C^{\circ}\right) w(C) \geqslant 1$ for every symmetric convex body $C$, and taking into account (3.6), we see that $w\left(Z_{q}^{\circ}(\mu)\right) \geqslant c / \sqrt{q}$ for every $2 \leqslant q \leqslant q_{*}(\mu)$, and thus

$$
k_{*}\left(Z_{q}^{\circ}(\mu)\right) \geqslant c_{5} n \frac{w^{2}\left(Z_{q}^{\circ}(\mu)\right)}{R^{2}\left(Z_{q}^{\circ}(\mu)\right)} \geqslant \frac{c_{6} n}{q} .
$$

3. Small ball probability estimates. Given a symmetric convex body $C$ in $\mathbb{R}^{n}$ we set $\bar{C}=|C|^{-1 / n} C$. In this last subsection we describe an approach which can lead to small ball probability estimates for the centroid bodies. It is convenient to normalize the volume and consider $\overline{Z_{q}}(\mu)$ instead of $Z_{q}(\mu)$. Recall that if $q \leqslant \sqrt{n}$, then $\left|Z_{q}(\mu)\right|^{1 / n} \simeq \sqrt{q / n}$, and hence

$$
\overline{Z_{q}}(\mu) \simeq \sqrt{n / q} Z_{q}(\mu) .
$$

Then, $w\left(\overline{Z_{q}}(\mu)\right) \simeq \sqrt{n / q} w\left(Z_{q}(\mu)\right) \simeq \sqrt{n}$, which implies

$$
\log N\left(\overline{Z_{q}}(\mu), s B_{2}^{n}\right) \leqslant c_{1} n\left(\frac{w\left(\overline{Z_{q}}(\mu)\right)}{s}\right)^{2} \leqslant \frac{c_{2} n^{2}}{s^{2}} .
$$


We use the following fact ([8], Lemma 5.6).

Lemma 8.8. Let $C$ be a centered convex body of volume 1 in $\mathbb{R}^{n}$. Assume that for some $s>0$,

$$
r_{s}:=\log N\left(K, s B_{2}^{n}\right)<n
$$

Then,

$$
I_{-r_{s}}(K) \leqslant c s .
$$

We apply Lemma 8.8 for $\overline{Z_{q}}(\mu)$ to get:

Proposition 8.9. Let $\mu$ be an isotropic log-concave measure on $\mathbb{R}^{n}$. If $2 \leqslant q \leqslant \sqrt{n}$, then

for all $1 \leqslant r \leqslant c n$.

$$
I_{-r}\left(\overline{Z_{q}}(\mu)\right) \leqslant \frac{c_{3} n}{\sqrt{r}}
$$

From Markov's inequality we have the small ball probability estimate

$$
\left|\left\{x \in \overline{Z_{q}}(\mu):\|x\|_{2} \leqslant \varepsilon I_{-r}\left(\overline{Z_{q}}(\mu)\right)\right\}\right| \leqslant \varepsilon^{r} .
$$

\section{ACKNOWLEDGEMENT}

The authors would like to acknowledge support from the program "API $\Sigma T E I A$ II-ATOCB-3566" of the General Secretariat for Research and Technology of Greece.

\section{REFERENCES}

[1] S. Artstein, V. Milman, and S. J. Szarek, Duality of metric entropy, Ann. of Math. (2) 159 (2004), no. 3, 1313-1328, DOI 10.4007/annals.2004.159.1313. MR2113023 (2005h:47037)

[2] Keith Ball, Logarithmically concave functions and sections of convex sets in $\mathbf{R}^{n}$, Studia Math. 88 (1988), no. 1, 69-84. MR932007 (89e:52002)

[3] Jesús Bastero, Upper bounds for the volume and diameter of $m$-dimensional sections of convex bodies, Proc. Amer. Math. Soc. 135 (2007), no. 6, 1851-1859 (electronic), DOI 10.1090/S0002-9939-07-08693-5. MR2286096 (2007m:46019)

[4] J. Bourgain, On the distribution of polynomials on high-dimensional convex sets, Geometric aspects of functional analysis (1989-90), Lecture Notes in Math., vol. 1469, Springer, Berlin, 1991, pp. 127-137, DOI 10.1007/BFb0089219. MR1122617 (92j:52007)

[5] J. Bourgain and V. D. Milman, New volume ratio properties for convex symmetric bodies in $\mathbf{R}^{n}$, Invent. Math. 88 (1987), no. 2, 319-340, DOI 10.1007/BF01388911. MR880954 (88f:52013)

[6] J. Bourgain, J. Lindenstrauss, and V. D. Milman, Minkowski sums and symmetrizations, Geometric aspects of functional analysis (1986/87), Lecture Notes in Math., vol. 1317, Springer, Berlin, 1988, pp. 44-66, DOI 10.1007/BFb0081735. MR950975 (89g:46025)

[7] Silouanos Brazitikos, Apostolos Giannopoulos, Petros Valettas, and Beatrice-Helen Vritsiou, Geometry of isotropic convex bodies, Mathematical Surveys and Monographs, vol. 196, American Mathematical Society, Providence, RI, 2014. MR3185453

[8] Nikos Dafnis and Grigoris Paouris, Small ball probability estimates, $\psi_{2}$-behavior and the hyperplane conjecture, J. Funct. Anal. 258 (2010), no. 6, 1933-1964, DOI 10.1016/j.jfa.2009.06.038. MR2578460 (2011a:60111)

[9] A. Giannopoulos, Notes on isotropic convex bodies, lecture notes, Warsaw, 2003, available at http://users.uoa.gr/ apgiannop/.

[10] A. A. Giannopoulos and V. D. Milman, On the diameter of proportional sections of a symmetric convex body, Internat. Math. Res. Notices 1 (1997), 5-19, DOI 10.1155/S1073792897000020. MR1426731 (97k:52003)

[11] A. A. Giannopoulos and V. D. Milman, Mean width and diameter of proportional sections of a symmetric convex body, J. Reine Angew. Math. 497 (1998), 113-139, DOI 10.1515/crll.1998.036. MR 1617429(99c:52006) 
[12] Apostolos A. Giannopoulos and Vitali D. Milman, Euclidean structure in finite dimensional normed spaces, Handbook of the geometry of Banach spaces, Vol. I, North-Holland, Amsterdam, 2001, pp. 707-779, DOI 10.1016/S1874-5849(01)80019-X. MR1863705 (2003b:46008)

[13] Apostolos Giannopoulos, Vitali D. Milman, and Antonis Tsolomitis, Asymptotic formulas for the diameter of sections of symmetric convex bodies, J. Funct. Anal. 223 (2005), no. 1, 86-108, DOI 10.1016/j.jfa.2004.10.006. MR2139881 (2006b:46010)

[14] A. Giannopoulos, G. Paouris, and P. Valettas, On the existence of subgaussian directions for log-concave measures, Concentration, functional inequalities and isoperimetry, Contemp. Math., vol. 545, Amer. Math. Soc., Providence, RI, 2011, pp. 103-122, DOI 10.1090/conm/545/10768. MR2858469 (2012k:52007)

[15] Apostolos Giannopoulos, Grigoris Paouris, and Petros Valettas, On the distribution of the $\psi_{2}$-norm of linear functionals on isotropic convex bodies, Geometric aspects of functional analysis, Lecture Notes in Math., vol. 2050, Springer, Heidelberg, 2012, pp. 227-253, DOI 10.1007/978-3-642-29849-3_13. MR2985135

[16] Apostolos Giannopoulos, Grigoris Paouris, and Beatrice-Helen Vritsiou, A remark on the slicing problem, J. Funct. Anal. 262 (2012), no. 3, 1062-1086, DOI 10.1016/j.jfa.2011.10.011. MR2863856(2012m:52011)

[17] Y. Gordon, On Milman's inequality and random subspaces which escape through a mesh in $\mathbf{R}^{n}$, Geometric aspects of functional analysis (1986/87), Lecture Notes in Math., vol. 1317, Springer, Berlin, 1988, pp. 84-106, DOI 10.1007/BFb0081737. MR.950977 (90b:46036)

[18] B. Klartag, On convex perturbations with a bounded isotropic constant, Geom. Funct. Anal. 16 (2006), no. 6, 1274-1290, DOI 10.1007/s00039-006-0588-1. MR2276540(2007i:52005)

[19] Bo'az Klartag and Emanuel Milman, Centroid bodies and the logarithmic Laplace transforma unified approach, J. Funct. Anal. 262 (2012), no. 1, 10-34, DOI 10.1016/j.jfa.2011.09.003. MR2852254

[20] Bo'az Klartag and Emanuel Milman, Inner regularization of log-concave measures and smallball estimates, Geometric aspects of functional analysis, Lecture Notes in Math., vol. 2050, Springer, Heidelberg, 2012, pp. 267-278, DOI 10.1007/978-3-642-29849-3_15. MR2985297

[21] B. Klartag and V. Milman, Rapid Steiner symmetrization of most of a convex body and the slicing problem, Combin. Probab. Comput. 14 (2005), no. 5-6, 829-843, DOI 10.1017/S0963548305006899. MR2174659 (2006j:52003)

[22] B. Klartag and R. Vershynin, Small ball probability and Dvoretzky's theorem, Israel J. Math. 157 (2007), 193-207, DOI 10.1007/s11856-006-0007-1. MR2342445 (2009b:46017)

[23] A. E. Litvak, V. D. Milman, and G. Schechtman, Averages of norms and quasi-norms, Math. Ann. 312 (1998), no. 1, 95-124, DOI 10.1007/s002080050213. MR1645952 (2000c:46013)

[24] A. E. Litvak, V. D. Mil'man, A. Pazhor, and N. Tomchak-Egermann, Entropy extension (Russian, with Russian summary), Funktsional. Anal. i Prilozhen. 40 (2006), no. 4, 65-71, 112, DOI 10.1007/s10688-006-0046-8; English transl., Funct. Anal. Appl. 40 (2006), no. 4, 298-303. MR2307703 (2008a:46011)

[25] Erwin Lutwak, Deane Yang, and Gaoyong Zhang, $L_{p}$ affine isoperimetric inequalities, J. Differential Geom. 56 (2000), no. 1, 111-132. MR1863023(2002h:52011)

[26] V. D. Milman, Isomorphic symmetrizations and geometric inequalities, Geometric aspects of functional analysis (1986/87), Lecture Notes in Math., vol. 1317, Springer, Berlin, 1988, pp. 107-131, DOI 10.1007/BFb0081738. MR950978 (89h:52011)

[27] V. Milman, Some applications of duality relations, Geometric aspects of functional analysis (1989-90), Lecture Notes in Math., vol. 1469, Springer, Berlin, 1991, pp. 13-40, DOI 10.1007/BFb0089213. MR1122611 (92h:52009)

[28] V. D. Milman and A. Pajor, Isotropic position and inertia ellipsoids and zonoids of the unit ball of a normed $n$-dimensional space, Geometric aspects of functional analysis (1987-88), Lecture Notes in Math., vol. 1376, Springer, Berlin, 1989, pp. 64-104, DOI 10.1007/BFb0090049. MR1008717 (90g:52003)

[29] Vitali D. Milman and Gideon Schechtman, Asymptotic theory of finite-dimensional normed spaces, Lecture Notes in Mathematics, vol. 1200, Springer-Verlag, Berlin, 1986. With an appendix by M. Gromov. MR $856576(87 \mathrm{~m}: 46038)$

[30] G. Paouris, Concentration of mass on convex bodies, Geom. Funct. Anal. 16 (2006), no. 5, 1021-1049, DOI 10.1007/s00039-006-0584-5. MR2276533 (2007k:52009)

[31] Grigoris Paouris, Small ball probability estimates for log-concave measures, Trans. Amer. Math. Soc. 364 (2012), no. 1, 287-308, DOI 10.1090/S0002-9947-2011-05411-5. MR2833584 
[32] Gilles Pisier, A new approach to several results of V. Milman, J. Reine Angew. Math. 393 (1989), 115-131, DOI 10.1515/crll.1989.393.115. MR972362(90a:46038)

[33] Gilles Pisier, The volume of convex bodies and Banach space geometry, Cambridge Tracts in Mathematics, vol. 94, Cambridge University Press, Cambridge, 1989. MR.1036275 (91d:52005)

[34] Rolf Schneider, Convex bodies: the Brunn-Minkowski theory, Encyclopedia of Mathematics and its Applications, vol. 44, Cambridge University Press, Cambridge, 1993. MR.1216521 (94d:52007)

[35] P. Valettas, Upper bound for the $\ell$-norm in the isotropic position, private communication.

[36] Roman Vershynin, Isoperimetry of waists and local versus global asymptotic convex geometries, with an appendix by Mark Rudelson and Vershynin, Duke Math. J. 131 (2006), no. 1, 1-16, DOI 10.1215/S0012-7094-05-13111-8. MR2219235 (2007h:46013)

[37] Beatrice-Helen Vritsiou, Further unifying two approaches to the hyperplane conjecture, Int. Math. Res. Not. IMRN 6 (2014), 1493-1514. MR3180599

Department of Mathematics, University of Athens, Panepistimioupolis 157 84, Athens, GREECE

E-mail address: apgiannop@math.uoa.gr

Department of Mathematics, University of Athens, Panepistimioupolis 157 84, Athens, Greece

E-mail address: pantstav@yahoo.gr

Department of Mathematics, University of the Aegean, Karlovassi 832 00, Samos, Greece

E-mail address: antonis.tsolomitis@gmail.com

Department of Mathematics, University of Athens, Panepistimioupolis 157 84, Athens, Greece

E-mail address: bevritsi@math.uoa.gr

Current address: Department of Mathematics, University of Michigan, Ann Arbor, Michigan 48109

E-mail address: vritsiou@umich.edu 